\title{
CRISPR-Cas Systems and the Paradox of Self-Targeting Spacers
}

\author{
Franziska Wimmer ${ }^{1}$ and Chase L. Beisel ${ }^{1,2 *}$ \\ ' Helmholtz Institute for RNA-Based Infection Research (HIRI), Helmholtz Centre for Infection Research (HZI), Würzburg, \\ Germany, ${ }^{2}$ Medical Faculty, University of Würzburg, Würzburg, Germany
}

CRISPR-Cas immune systems in bacteria and archaea record prior infections as spacers within each system's CRISPR arrays. Spacers are normally derived from invasive genetic material and direct the immune system to complementary targets as part of future infections. However, not all spacers appear to be derived from foreign genetic material and instead can originate from the host genome. Their presence poses a paradox, as self-targeting spacers would be expected to induce an autoimmune response and cell death. In this review, we discuss the known frequency of self-targeting spacers in natural CRISPR-Cas systems, how these spacers can be incorporated into CRISPR arrays, and how the host can evade lethal attack. We also discuss how

OPEN ACCESS

Edited by:

David S. Weiss,

Emory University, United States

Reviewed by:

Nan Peng,

Huazhong Agricultural University,

China

Yuqing $\mathrm{Li}$,

Sichuan University, China

*Correspondence:

Chase L. Beise

chase.beise/@helmholtz-hiri.de

Specialty section:

This article was submitted to

Microbial Physiology and Metabolism,

a section of the journal

Frontiers in Microbiology

Received: 01 September 2019

Accepted: 19 December 2019

Published: 22 January 2020

Citation:

Wimmer F and Beisel CL (2020) CRISPR-Cas Systems

and the Paradox of Self-Targeting

Spacers. Front. Microbiol. 10:3078.

doi: 10.3389/fmicb.2019.03078 self-targeting spacers can become the basis for alternative functions performed by CRISPR-Cas systems that extend beyond adaptive immunity. Overall, the acquisition of genome-targeting spacers poses a substantial risk but can aid in the host's evolution and potentially lead to or support new functionalities.

Keywords: CRISPR-Cas, spacer acquisition, anti-CRISPR proteins, autoimmunity, gene regulation

\section{INTRODUCTION}

CRISPR-Cas systems represent highly diverse adaptive immune systems found in many bacteria and most archaea (Barrangou et al., 2007; Sorek et al., 2013; Koonin et al., 2017). These systems consist of two general parts: Clustered Regularly Interspaced Short Palindromic Repeats (CRISPR) arrays and CRISPR-associated (Cas) proteins. CRISPR arrays represent the immunological memory of prior infections encoded within individual spacers separated by conserved repeats. Cas proteins carry out the adaptive immune functions. The Cas proteins are highly diverse, resulting in CRISPRCas systems currently being grouped into two classes, six types, and over 30 subtypes (Makarova et al., 2015; Koonin et al., 2017; Koonin and Makarova, 2019).

While the specific proteins and biomolecular mechanisms vary, all systems act through three general steps as part of adaptive immunity. The first step, acquisition, incorporates pieces of invading nucleic acids, called protospacers, as new spacers within the CRISPR array. The protospacers are often selected based on the presence of a flanking protospacer adjacent motif (PAM) (Yosef et al., 2013; Wang et al., 2015). Acquisition requires the universal Cas proteins Cas1 and Cas2 (Yosef et al., 2012; Nuñez et al., 2014), although other accessory factors such as Cas4 (Kieper et al., 2018), Csal (Liu T. et al., 2017), Csn2 (Heler et al., 2015; Wei et al., 2015) and reverse transcriptase (RT) (Kojima and Kanehisa, 2008; Simon and Zimmerly, 2008; Silas et al., 2016) can also be involved. In type II CRISPR-Cas systems, the effector nuclease Cas 9 can also play an essential 
role in the acquisition of new spacers (Heler et al., 2015; Wei et al., 2015). The acquired spacers serve as DNA records of prior infections that are passed to the host's progeny.

The second and third steps involve the biogenesis of CRISPR RNAs (crRNAs) from the CRISPR arrays followed by crRNAdirected immune defense. As part of crRNA biogenesis, the CRISPR array is, for most cases, transcribed into a long precursor CRISPR RNA (pre-crRNA) and processed into mature crRNAs by Cas proteins. In some cases, processing involves accessory factors such as RNase III (Carte et al., 2008; Deltcheva et al., 2011; Behler et al., 2018; Lee et al., 2018, 2019). The crRNA then forms a complex with Cas effector proteins to target foreign nucleic acids. Class 2 CRISPR-Cas systems rely on only one protein to bind and cleave their targets, with type II systems and some type $\mathrm{V}$ systems also requiring a trans-activating crRNA (tracrRNA) for effector complex formation (Deltcheva et al., 2011; Shmakov et al., 2015; Zetsche et al., 2015). Class I systems in contrast rely on multiple proteins that form a multi-subunit effector complex (Brouns et al., 2008; Hale et al., 2009). The resulting ribonucleoprotein complex then surveils the host's cytoplasm for DNA and/or RNA sequences that are complementary to the spacer and flanked either by a PAM or a sequence lacking complementarity to the corresponding portion of the crRNA repeat (Mojica et al., 2005; Marraffini and Sontheimer, 2010; Leenay and Beisel, 2017; Meeske and Marraffini, 2018).

One commonality across CRISPR-Cas systems is their reliance on the array-encoded spacers to direct CRISPR-based immunity. To date, only 1-19\% of identified spacers have been matched to potential protospacer sites, where most of the assigned spacers appear to be derived from the genome of bacteriophages (herein called phages), archaeal viruses (herein called viruses), plasmids or other organisms (Bolotin et al., 2005; Mojica et al., 2005; Pourcel et al., 2005; Marraffini and Sontheimer, 2008; Brodt et al., 2011; Bikard et al., 2012; Shmakov et al., 2017). However, many of the assigned spacers match sequences within the host genome, what are generally called self-targeting spacers.

Self-targeting spacers are unexpected due to an observed preference toward acquiring foreign genetic material (Levy et al., 2015) and heavy cytotoxicity to the host because self-targeting of the host's chromosome would lead to cell death (Stern et al., 2010; Jiang et al., 2013; Vercoe et al., 2013; Gomaa et al., 2014; Figure 1A). Here, we review the presence and consequences of self-targeting spacers. We address the known distribution of self-targeting spacers in sequenced CRISPR-Cas systems. We then discuss different mechanisms of acquisition that could generate self-targeting spacers and how these organisms can survive despite the potential for chromosomal targeting and autoimmunity. Finally, we report some of the beneficial functions that have been associated with the self-targeting spacers that can imbue CRISPR-Cas systems with functionalities that extend beyond adaptive immunity. This content greatly expands on an earlier mini-review on the consequences of chromosomal targeting (Heussler and O'Toole, 2016) and incorporates recently reported examples of self-targeting reflecting alternative functions of these prevalent adaptive immune systems.

\section{NATURAL OCCURRENCE OF SELF-TARGETING SPACERS}

Multiple studies have explored the source of spacers in diverse CRISPR-Cas systems, with recurring observations of self-targeting spacers. In the first broad study of matching protospacers, 88 of the analyzed 4,500 spacers were similar to known sequences, and $35 \%$ of these spacers matched chromosomal DNA not directly related to foreign genetic elements (Mojica et al., 2005). Separately, a study from 2008 found that $7 \%$ of spacers in different CRISPR-Cas systems from Streptococcus thermophilus matched chromosomal sequences (Horvath et al., 2008). One year later, the same group analyzed CRISPR-Cas systems from a more diverse set of lactic acid bacteria, reporting that 23 of the 104 spacers matched the chromosome (Horvath et al., 2009). Shortly thereafter, one study analyzed the CRISPR arrays of the 330 prokaryotes containing CRISPR-Cas systems included in the CRISPRdb database (Grissa et al., 2007) in 2010, with self-targeting spacers comprising $0.4 \%$ of all spacers (including the vast majority of spacers with no assignable protospacers) and appearing in $18 \%$ of the included prokaryotic genomes (Stern et al., 2010).

The number of sequenced organisms has increased over time, allowing more recent studies to more deeply and widely interrogate spacer origins. For instance, one study in 2017 screened $\sim 50,000$ completely or partially assembled genomes, while another study in 2018 used the online tool CRISPRminer to evaluate more than 60,000 organisms harboring a CRISPR array (Shmakov et al., 2017; Zhang et al., 2018). Shmakov et al. assigned protospacer locations to $7 \%$ of the detected 363,460 unique spacers, with $\sim 6 \%$ of these spacers matching prokaryotic genomes and $16 \%$ of these genome-matching spacers being potentially unrelated to (pro-)viral sequences (Shmakov et al., 2017). The study with CRISPRminer reported 22,110 selftargeting events in publications (Stern et al., 2010; Rauch et al., 2017; Watters et al., 2018) and could predict 6,260 additional putative self-targeting spacers in 4,136 organisms, implying that $\sim 7 \%$ of the genomes within their database should harbor at least one self-targeting spacer (Zhang et al., 2018).

The natural acquisition of self-targeting spacers has also been observed as part of adaptive evolution studies between phages and their prokaryotic host. Two key studies relied on a strain of the bacterium $S$. thermophilus harboring two type II-A CRISPRCas systems (Paez-Espino et al., 2013, 2015). In these studies, only $0.01-0.04 \%$ of the observed new spacers matched the genome. These frequencies are lower than those reported in the largescale bioinformatics studies, although this discrepancy can be attributed in part to the selective pressure exerted by the actively infecting phages.

\section{INCORPORATION OF SELF-TARGETING SPACERS}

Given the frequency of self-targeting spacers and their potential for autoimmunity, we next discuss the circumstances under which a self-targeting spacer can be acquired. In particular, we 


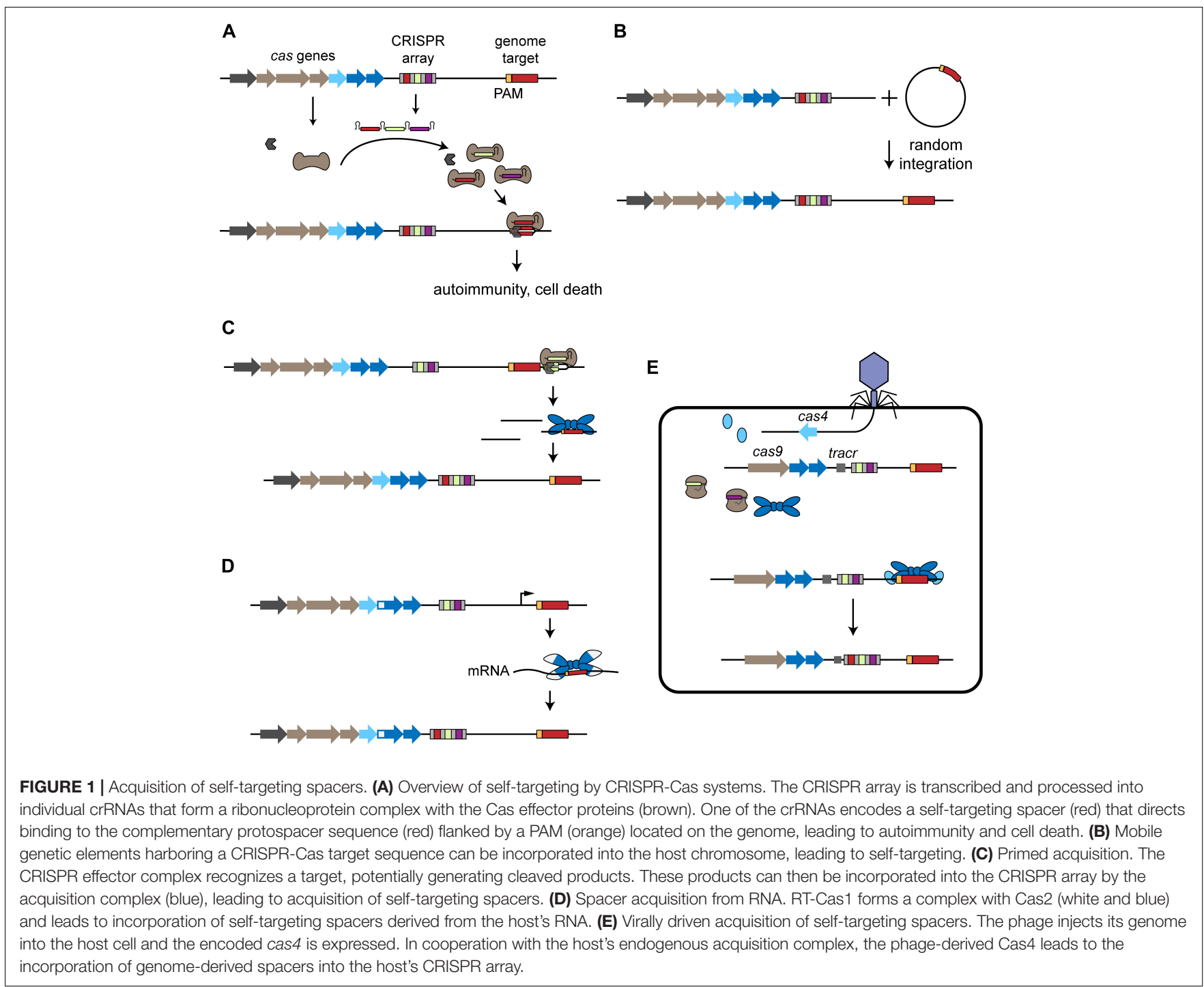

consider five general scenarios that have been reported: naïve acquisition from DNA, protospacers within a transferred mobile genetic element (MGE), primed adaptation, naive acquisition from RNA, and phage/virus-triggered acquisition from host DNA. For many of these scenarios, we address the extent to which acquisition differentiates between chromosomal and foreign genetic material and the known associated mechanisms. We finally must note that our understanding of CRISPR-based acquisition is still developing, and other mechanisms within the diversity of CRISPR-Cas systems likely await discovery.

\section{Naïve Acquisition}

Naive acquisition leads to the incorporation of new spacers without any influence from the existing pool of spacers. Cas1 and Cas2 are required while Cas4, Csn2 or Cas9 may be additionally needed depending on the system sub-type (Yosef et al., 2012; Nuñez et al., 2014; Heler et al., 2015; Wei et al., 2015; Kieper et al., 2018). It was known for many years that protospacers were commonly flanked by PAMs to allow targeting by the effector proteins to differentiate between self and non-self targets (Deveau et al., 2008; Horvath et al., 2008). However, it remained unclear how the acquisition machinery differentiated between invader and chromosomal DNA. In one of the first studies to systematically interrogate spacer acquisition, Levy and coworkers sequenced over 38 million newly acquired spacers following plasmid-based expression of Cas1 and Cas2 in an Escherichia coli strain harboring a CRISPR array but lacking endogenous cas genes. They found that spacers were preferentially acquired from replication forks, presumably due to stalling during replication and degradation by RecBCD. This preference resulted in 100fold to 1,000-fold enrichment of spacers derived from a resident plasmid compared to the chromosome. The high-copy number plasmids present most of the replication forks in a replicating cell, partly explaining the preference toward high copy plasmids (Levy et al., 2015).

Another critical factor identified by Levy and coworkers was the presence of Chi sites. These sequence motifs interact with and prevent DNA degradation by RecBCD (Smith, 2012) at the 
sites of double-stranded DNA breaks that often occur at stalled replication forks (Kuzminov, 2001; Michel et al., 2001). Due to the fact that Chi sites occur approximately every $5 \mathrm{~kb}$ in the E. coli genome (El Karoui et al., 1999), these Chi sites were hypothesized to mark the host DNA as "self" and prevent acquisition of spacers from the host's genome. The plasmids contained fewer Chi sites, likely further contributing to preferential acquisition from this DNA. Linear viral DNA would also offer a preferred substrate for RecBCD, resulting in DNA fragments that can be used to generate new spacers (Levy et al., 2015). Phages are known to encode RecBCD inhibitors and some also encode a large number of Chi sites in their genome (Friedman and Hays, 1986; Murphy and Lewis, 1993; Bobay et al., 2013), thus potentially countering acquisition by CRISPR-Cas systems.

Liu et al. observed a different element influencing naïve acquisition in Sulfolobus islandicus, an archaeon that encodes one type I-A CRISPR-Cas system and two type III-B CRISPRCas systems. Following overexpression of Csa3a that drives expression of the type I-A acquisition genes, S. islandicus integrated spacers from the csa3a expression plasmid as well as from its own genome with a high bias toward the plasmid (Liu et al., 2015). Interestingly, for deletion mutants lacking RNA processing or nuclease activity, $<28 \%$ of spacers were derived from the plasmid (Liu T. et al., 2017). While this fraction was far less than the $>90 \%$ in a previous study (Liu et al., 2015), it still reflected preferential acquisition from plasmids when taking into account the relative length of the plasmid and chromosomal DNA (Liu T. et al., 2017). The stronger preference for plasmid DNA in the presence of an active CRISPR-Cas system may be explained in part by the cytotoxicity of genome targeting by the active but not impaired system upon self-targeting.

Spacer acquisition in type II CRISPR-Cas systems also appears to differ for active versus impaired CRISPR-Cas systems. Wei et al. (2015) looked at acquisition requirements in a type IIA CRISPR-Cas system by expressing the different CRISPR-Cas components on plasmids and monitoring spacer acquisition. They found that acquisition required the presence of Cas9, in contrast to spacer acquisition by Cas1 and Cas 2 in type I CRISPR-Cas systems. Interestingly, the authors found that the cleavage activity of Cas 9 contributed to an observed preference for acquisition from plasmid DNA. Specifically, by using a mutated Cas 9 that disrupts its cleavage activity (dCas9), the authors shifted the fraction of plasmid-derived spacers from $68 \%$ to $4 \%$, representing a loss of preference given the matching ratio of plasmid DNA to genomic DNA (Wei et al., 2015). In total, naïve acquisition by different types of CRISPR-Cas systems can lead to the incorporation of self-targeting spacers, although foreign genetic material is the predominant source of spacers. It would be interesting to investigate if the above reported phenomena can also be observed in different organisms or other CRISPR-Cas systems that rely on additional Cas proteins for acquiring new spacers.

\section{Protospacers Within Transferred Mobile Genetic Elements}

Many self-targeting spacers identified in nature bear homology to MGEs such as transposons or prophages/proviruses that have been incorporated into the genome. These spacers could have been acquired prior to the incorporation of the MGE as a preventative measure, or afterward to induce cell death and prevent further spread of the MGE (Figure 1B). All evidence of this mechanism comes from bioinformatic experiments. Looking at self-targeting spacers with $100 \%$ complementary to their predicted protospacer region, Stern et al. (2010) found an approximately equal distribution of protospacers from mobile elements encoded in the chromosome and non-mobile elements (47\% vs. 53\%). In comparison, Shmakov et al. (2017) assigned $83 \%$ of the self-targeting spacers to (pro-)phage sequences. The difference might arise from the greater abundance of sequenced MGEs over time (Geer et al., 2010; Akhter et al., 2012). Nevertheless, these frequencies leave ample spacers derived from non-mobile elements.

\section{Primed Adaptation}

A different potential means of incorporating self-targeting spacers is through primed adaptation (or primed acquisition). Acquisition of spacers under primed adaptation requires target recognition with pre-existing spacers that are partially or fully complementary to the foreign DNA. Recognition leads to the acquisition of multiple spacers from sites in close proximity to the existing protospacer (Datsenko et al., 2012; Swarts et al., 2012; Richter et al., 2014; Jackson et al., 2019; Figure 1C).

Bioinformatic evidence indicates that primed adaptation is widespread in type I and type II CRISPR-Cas systems (Nicholson et al., 2019). Primed adaptation by type I systems involves degradation of the target site by Cas3 and incorporation of the degradation products as new spacers by Cas1 and Cas2 (Künne et al., 2016). Primed adaptation by type II systems is not well understood, although Nicholson et al. (2019) proposed two possible pathways: one that involves a main role of Cas9, and another involving host-specific processes such as DNA repair producing pre-spacers at the sites of target cleavage.

Regardless of the exact mechanism, primed adaptation is expected to preferentially incorporate foreign genetic material due to the pre-existence of more spacers derived from nonchromosomal elements. However, primed acquisition of host DNA could occur upon targeting MGE that were incorporated into a bacterial or archaeal genome (Nicholson et al., 2019). Primed acquisition outside of the borders of the MGE could also be triggered, leading to incorporation of non-mobile self DNA from the chromosome. Finally, spacers that evolved to target foreign DNA might prime with similar sequences in chromosomal DNA (Staals et al., 2016), where prior work showed that priming can occur even with 13 mutations in the target site relative to the pre-existing spacer (Fineran et al., 2014).

\section{Naïve Acquisition of RNA-Derived Spacers}

One unique mode of acquisition is through relatively rare Cas proteins that recognize RNA rather than DNA. These proteins include a RT often translationally fused to Cas1 or to a fusion between Cas 1 and the Cas6 protein responsible for crRNA biogenesis. This unique RNA-acquiring machinery is predominantly associated with type III CRISPR-Cas systems 
but is also found with type I-E and type VI-A systems (Kojima and Kanehisa, 2008; Simon and Zimmerly, 2008; Toro and NisaMartínez, 2014; Silas et al., 2017; Toro et al., 2019a,b). For the few examples that have been studied, these RTs reversetranscribe an acquired RNA into DNA to produce a substrate for acquisition (Silas et al., 2016). If the RNA-derived spacers are derived from host RNA, the associated type III CRISPRCas systems can now target the host and lead to autoimmunity (Figure 1D). Interestingly, self-targeting spacers have been found in three strains encoding a RT as part of their type III CRISPR-Cas systems (Silas et al., 2017; Zhang et al., 2018).

Other systems solely encode RT and Cas1 and lack all other Cas proteins, holding the potential to acquire self-targeting spacers without inducing autoimmunity. As one example, Rivularia sp. PCC 7116 encodes Cas1, Cas2, and RT in a distinct genomic island compared to the other CRISPR-Cas systems present in that bacterium. The CRISPR array associated with Cas1, Cas2 and RT harbors a spacer matching a hypothetical gene encoded on the bacterial chromosome (Silas et al., 2017; Kersey et al., 2018; Zhang et al., 2018). The lack of effector proteins suggests that these systems are used for alternative functions rather than immunity, although this has not been investigated to-date.

The unique sourcing of spacers from RNA raises questions about how the acquisition machinery selected some RNA sequences over others. Silas and coworkers sequenced the spacer content in an open-air culture of Arthrospira platensis, which encodes a RT-Cas1 fusion as part of its type IIIB CRISPR-Cas system (Silas et al., 2017). Most of the associated protospacers could not be identified, and the few that could be identified traced to DNA viruses. Schmidt and coworkers were able to gain more extensive insights by monitoring spacer acquisition in $E$. coli following plasmidbased expression of the type III Fusicatenibacter saccharivorans RT-Cas1 and Cas2. While spacers were derived from RNAs encoded in the chromosome and plasmid, there was a strong preference for A/T-rich sequences at the ends of highly expressed genes. Interestingly, there was no obvious preference for a flanking motif or for plasmid-encoded RNAs (Schmidt et al., 2018). Further studies are needed to fully understand preferences exhibited by type III CRISPR-Cas systems for RNA acquisition.

\section{Acquisition of Self-Targeting Spacers Triggered by Foreign Invaders}

There is also evidence that phages can encode Cas proteins that drive endogenous CRISPR-Cas systems to preferentially acquire self-targeting spacers. The first direct evidence comes from studying the origin of spacers encoded within the CRISPR array of Campylobacter jejuni PT14 harboring a minimal type II-C CRISPR-Cas system (Hooton and Connerton, 2014). While the spacers do not share $100 \%$ sequence identity with any known sequences, some of the spacers partially matched chromosomal sequences in the PT14 genome. Tracking spacer content in a co-culture of PT14 cells and CP8/CP30A phage revealed that all newly acquired spacers were derived from the host's chromosome and not the phage (Hooton and Connerton, 2014). The phage encoded a copy of the cas 4 gene involved in protospacer maturation as part of many CRISPR-Cas systems (Zhang et al., 2012; Lemak et al., 2013; Kieper et al., 2018; Lee et al., 2018), while the endogenous type II-C CRISPR-Cas system normally lacks this gene. The authors therefore attributed the unexpected selftargeting acquisition events to the phage encoded Cas4 (Hooton and Connerton, 2014; Figure 1E).

Further evidence that viral Cas 4 can impact host acquisition was found in S. islandicus. Zhang and coworkers evaluated the impact of the viral cas 4 gene found in a Sulfolobus spindle-shaped virus by transforming a plasmid encoding the viral cas 4 into S. islandicus. Cells harboring the plasmid exhibited less frequent spacer acquisition, although the frequency of spacers acquired from the plasmid or chromosome did not change. Furthermore, overexpression of host Cas 4 from a plasmid also led to reduced spacer acquisition. These findings suggest that overproduction of Cas4 can in some cases disable spacer acquisition. One explanation is that the viral encoded Cas4 serves as an antiCRISPR protein (Acr) by preventing spacer acquisition and in turn enabling escape from CRISPR-Cas targeting (Zhang et al., 2019). While more work is needed to elucidate the underlying role of the virally encoded Cas4, these examples and the many other instances of virally encoded Cas4 (Krupovic et al., 2015; Hudaiberdiev et al., 2017) suggest the intriguing possibility that phages and viruses could be actively directing the acquisition of spacers.

\section{SURVIVING SELF-TARGETING BY CRISPR-CAS SYSTEMS}

Unrelated to how prokaryotes incorporate spacers that target their own genome, cells must overcome self-targeting by their own CRISPR-Cas system to survive. CRISPR-based interference against the host's own genome is expected to lead to lethal autoimmunity due to the nuclease cutting within or close to their target site. Repair mechanisms in prokaryotes are often not efficient enough to fix CRISPR-Cas induced DNA damage, and DNA breaks often result in cell death (Stern et al., 2010; Jiang et al., 2013; Vercoe et al., 2013; Gomaa et al., 2014). Nevertheless, many different examples exist in which a self-targeting spacer can be tolerated. Below we describe each known mechanism.

\section{Active DNA Repair}

CRISPR-based targeting would be expected to induce irreparable damage, lest the cells repair invading genetic material and allow an infection to persist. Accordingly, many studies have reported that chromosomal targeting by CRISPR nucleases is cytotoxic in different bacteria and archaea (Stern et al., 2010; Jiang et al., 2013; Vercoe et al., 2013; Bikard et al., 2014; Citorik et al., 2014; Gomaa et al., 2014; Li Y. et al., 2016). That said, there exist examples in which intrinsic DNA repair mechanisms such as homologydirected repair (HDR), non-homologous end joining (NHEJ), and alternative end-joining (A-EJ) mechanisms allow cell survival (Chayot et al., 2010; Tong et al., 2015; Cui and Bikard, 2016; Stachler et al., 2017; Figure 2A). 


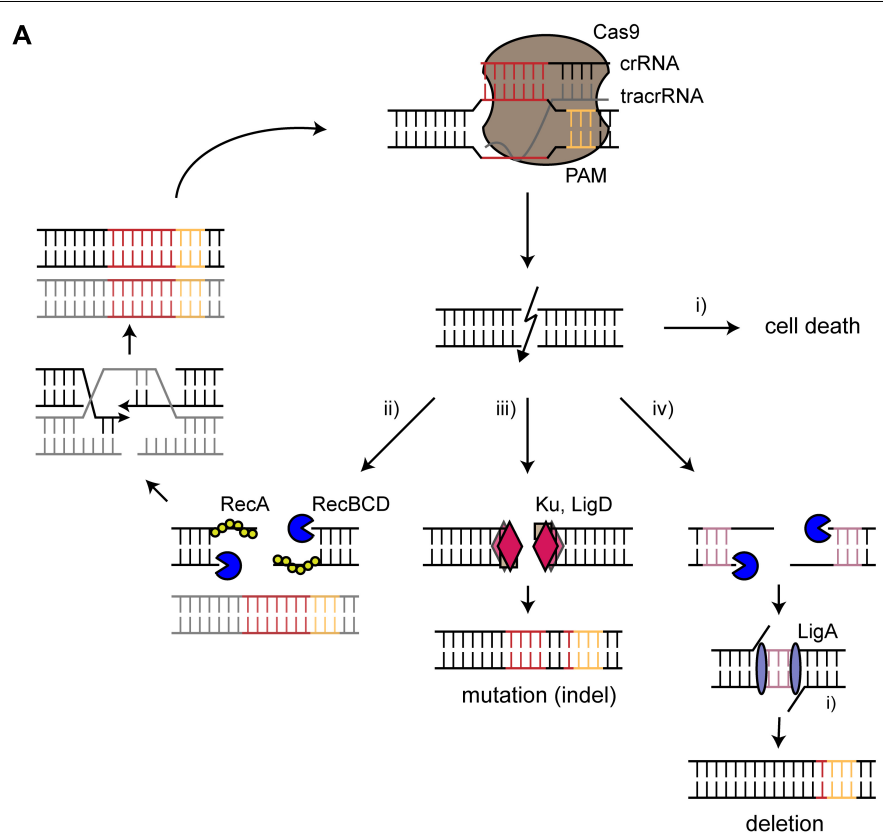

B
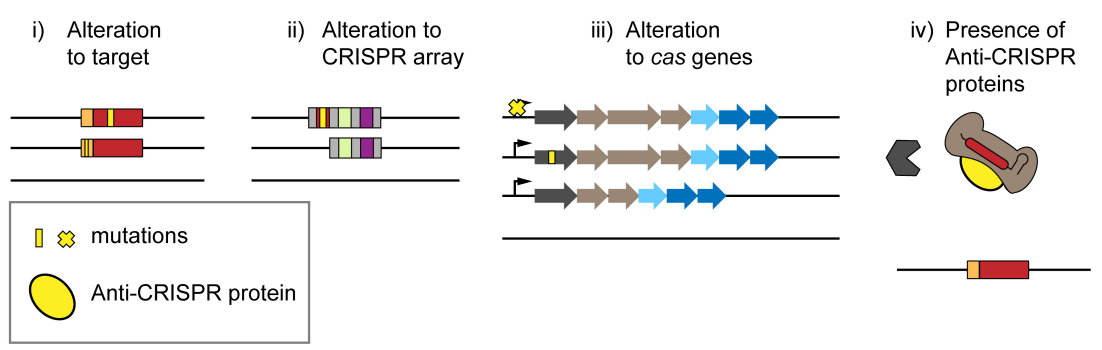

FIGURE 2 | Surviving self-targeting. (A) Survival of CRISPR-Cas targeting by intrinsic repair mechanisms. The type II effector protein Cas9 is used as an example. The CRISPR effector complex binds to its target in the genome (red) next to a PAM (orange), leading to a double-stranded break (DSB) causing different outcomes. (i) Cell death occurs if the break is not repaired. (ii) Homology-directed repair (HDR) restores the target site in the presence of an intact copy of the chromosome. DNA ends of the DSB undergo trimming in a Rec-dependent manner. HDR leads to the restoration of a chromosome that undergoes further attack by the CRISPR-Cas system. (iii) Non-homologous end joining leads to the formation of an insertion or deletion (indel). End joining is mediated by the repair proteins Ku and LigD. (iv) Alternative end-joining leads to deletions. DNA ends are trimmed by RecBCD until micro-homologous regions (purple) are reached. These regions are then ligated by LigA, resulting in deletions. (B) Escape from autoimmunity through mutations, deletions or active inhibition. (i,ii) mutations (yellow) or deletions within the protospacer, PAM or CRISPR array disrupts self-targeting. (iii) Mutation of the cas operon, inhibition of Cas expression or deletion of a cas gene or the entire locus can also prevent self-targeting. (iv) Anti-CRISPR proteins encoded within an integrated prophage can block CRISPR-Cas interference through different mechanisms, such as binding the Cas effector protein to prevent PAM recognition.

One potential mechanism is HDR through an additional copy of the chromosome. Cui and Bikard first observed this phenomenon when evaluating the consequences of targeting the E. coli chromosome with heterologously expressed Cas9 (Cui and Bikard, 2016). They found that targeting different sites within non-essential genes resulted in RecA-mediated HDR. Targeting did induce the SOS DNA-damage response, although the cells maintained their viability. In a separate example, Stachler and coworkers reported that the archaeon Haloferax volcanii could tolerate chromosomal targeting through its endogenous type I-B CRISPR-Cas system (Stachler et al., 2017). However, the tolerance could be attributed in part to the endogenous CRISPR array providing most of the crRNAs in the effector complexes. Deleting the Cas6 processing protein and expressing a mature self-targeting crRNA resulted in a fitness defect that was strengthened by expressing the crRNA at higher levels. The extent of self-targeting in the presence of the endogenous CRISPR array therefore was sufficiently weak to allow repair through HDR and the roughly 20 copies of the $H$. volcanii genome (Zerulla et al., 2014; Stachler et al., 2017). In both of these examples, there would likely be some selective pressure to disrupt self-targeting given the need for continuous repair.

Non-homologous end joining and alternative end-joining offer distinct repair mechanisms that permanently alter the target site, preventing further attack by CRISPR-Cas systems. NHEJ does not utilize a repair template and instead repairs double-stranded DNA breaks (DSBs) by adding insertions or deletions (indels) to the site of the DSB. Some prokaryotes 
possess relatively unsophisticated NHEJ machinery compared to eukaryotes, typically comprised of the complexes $\mathrm{Ku}$ and LigD (Aravind and Koonin, 2001; Weller et al., 2002; Gong et al., 2005; Bowater and Doherty, 2006; Shuman and Glickman, 2007; Tong et al., 2015). Some bacteria such as E. coli lacking $\mathrm{Ku}$ and LigD can utilize phage ligases to mediate NHEJ-like repair of CRISPR-Cas induced DSBs (Su et al., 2019). While NHEJ efficiently repairs DNA cleaved by some CRISPR nucleases in eukaryotic cells, some CRISPR-Cas induced DNA damage in prokaryotes is still highly cytotoxic when NHEJ is active (Xu et al., 2015; Bernheim et al., 2017). A-EJ is a repair mechanism that relies on microhomology-mediated end joining and largely leads to deletions. DSBs induce extensive endresection that is mostly dependent on RecBCD, while Ligase-A repairs the break by joining micro-homologous regions of 1 to 9 bps (Chayot et al., 2010; Figure 2A). Prior work suggested that A-EJ led to large deletions following genomic attack by a type I-F CRISPR-Cas system in Pectobacterium atrosepticum (Vercoe et al., 2013). Whether repair occurs through NHEJ or A-EJ, the resulting genome would be less susceptible (or even completely unsusceptible) to follow-up attack through the selftargeting spacer.

\section{Mutations Disrupting CRISPR-Based Targeting}

Mutations can prevent efficient CRISPR targeting in multiple ways. One way is mutation of the target site such as through NHEJ or A-EJ, impacting spacer complementarity or PAM recognition (Figure 2B). Two studies evaluating self-targeting through type II-A systems in S. thermophilus reported not only mutations of the targeted lac $Z$ gene but also deletion of the gene (Selle et al., 2015; Cañez et al., 2019). In one of the studies assessing self-targeting in the S. thermophilus strain LMD-9, targeting resulted in loss of $\sim 1.2 \mathrm{~kb}$ that included the lacZ gene. These deletions appeared to arise via genomic island excision via recombination between two flanking insertionsequence elements (Selle et al., 2015) that occur quite frequently in S. thermophilus (Bolotin et al., 2004). In contrast, another study reported an $\sim 40-\mathrm{kb}$ deletion upon targeting lac $Z$ in the S. thermophilus strain DGCC7710 that shares $99.2 \%$ sequence homology to LMD-9. No insertion-sequence elements could be detected within $50 \mathrm{~kb}$ flanking the lac $Z$ gene, potentially explaining why the same escape mechanism observed in LMD9 did not take place in DGCC7710. Recombination here might have happened between two regions encoding two galE genes sharing $86 \%$ nucleotide identity located $3 \mathrm{~kb}$ upstream and $30 \mathrm{~kb}$ downstream of lacZ (Cañez et al., 2019). One interesting possibility is that these large deletions existed in a small fraction of the cell population, where CRISPR-based targeting allowed this sub-population to survive (Selle et al., 2015; Cañez et al., 2019). The different outcomes of self-targeting in S. thermophilus LMD9 and DGCC7710 highlight the different escape mechanisms that can occur even between strains of the same species.

Escape from lethal self-targeting can not only occur via target mutation but also via mutations or deletions within the CRISPR array or the cas genes (Figure 2B). In the same study noted above (Cañez et al., 2019), the authors also investigated the escape mechanism by targeting lac $Z$ with the endogenous type I-E CRISPR-Cas system in S. thermophilus DGCC7710. Surprisingly, no deletions in the target site could be observed, and escape mutants consistently harbored defective plasmids missing the targeting spacer and one repeat likely caused by recombination between repeats that eliminated the self-targeting spacer (Cañez et al., 2019; Figure 2B). Thus, escape mechanisms can differ not only between strains but also between CRISPR-Cas systems. Loss of the plasmid-encoded spacer also occurred as the principal mode of escape when targeting E. coli's genome with its endogenous type I-E system (Gomaa et al., 2014). Separately, as an example of disrupting cas genes, Lactobacillus acidophilus NCFM appears to have deleted its entire cas gene cassette to avoid lethal self-targeting by six genome-targeting spacers encoded in the CRISPR array (Stern et al., 2010; Kersey et al., 2018; Zhang et al., 2018). Furthermore, mutations in the cas genes were reported when targeting the staphylococcal cassette chromosome mec (SSCmec) in Staphylococcus aureus through the endogenous III-A CRISPR-Cas system (Guan et al., 2017) or when targeting different sites through the I-F CRISPR-Cas system native to P. atrosepticum (Dy et al., 2013). Between disruption of the spacer or cas genes, explicit loss of an endogenous self-targeting spacer has been less reported in natural systems. However, this can be explained by bioinformatic searches having difficulties detecting loss of self-targeting spacers in genome databases given that the rest of the CRISPR array may still be intact.

Self-targeting by a CRISPR-Cas system also does not need to drive only one mode of escape. For instance, in the example of self-targeting through the type III-A CRISPR-Cas system in $S$. aureus, the authors reported different mutations or deletions in the escape mutants (Guan et al., 2017). Large deletions that included the target site occurred in $\sim 90 \%$ of the escape mutants, while spacer mutations or loss-of-function mutations in cas genes were also detected. Separately, in the example of self-targeting through the type I-F CRISPR-Cas system in P. atrosepticum, the bacterium harbors one naturally occurring self-targeting spacer that is not cytotoxic due to a mutation in the target's PAM (Dy et al., 2013). Transformation of plasmids harboring other selftargeting spacers further led to different sized deletions of regions containing the protospacer or removal of the cas operon. The frequency of one escape mode over another likely depends on different factors such as the frequency of background mutation and recombination, the types of mutations that can form, and the fitness defect that they introduce.

\section{Partially Complementary Spacers Directing Target Binding but Not Cleavage}

Mutations to the target site or the spacer can result in partial complementarity between spacers and their protospacers. For some systems, partial complementarity eliminates target cleavage but can preserve target binding. Comparison between off-target binding by dCas 9 and off-target cleavage by Cas 9 demonstrated extensive off-target binding but not cleavage (Wu et al., 2014). Another study also showed that partial target complementarity 
could allow an active Cas9 to bind but not cleave DNA, resulting in transcriptional silencing (Bikard et al., 2013). Wu and coworkers proposed a model which would explain the higher specificity of Cas9 by taking binding at the seed region into consideration. They hypothesized that PAM recognition by the Cas9:crRNA complex leads to DNA melting and enables base pairing between the spacer and the complementary seed region. As long as complementarity exists through the seed region, partial base pairing can allow target binding without cleavage (Wu et al., 2014). As a result, organisms could harbor spacers with partial complementarity to their own genome that would still drive target recognition but not autoimmunity.

\section{RNA Targeting}

While we have focused on CRISPR-Cas systems that explicitly target DNA, the type III and VI systems naturally target RNA as part of immune defense (Hale et al., 2009, 2012; Abudayyeh et al., 2016), with distinct implications for selftargeting. Type III CRISPR-Cas systems are capable of targeting DNA and RNA. The system's Csm or Cmr effector complex is guided to RNA targets complementary to the crRNA, triggering the sequence-specific RNase activity of Csm3 or Cmr4, respectively (Benda et al., 2014; Goldberg et al., 2014; Tamulaitis et al., 2014; Samai et al., 2015). Lack of complementarity between the $5^{\prime}$ crRNA handle and the target RNA activates single-stranded DNase activity by Cas10 (Jung et al., 2015; Kazlauskiene et al., 2016), although there is evidence of a $3^{\prime}$ RNA PAM motif that suggests diverging criteria for target selection across type III systems (Elmore et al., 2016). Furthermore, target recognition by the type III effector complex triggers Cas10 to produce cyclic adenylates. These molecules in turn activate the CRISPR accessory protein Csm6/Csx1, leading to non-specific RNA degradation to assist in viral defense (Kazlauskiene et al., 2017; Niewoehner et al., 2017; Rouillon et al., 2018).

In contrast to type III CRISPR-Cas systems, type VI systems represent the only systems known to-date that exclusively target RNA (Abudayyeh et al., 2016). Cas13, the type VI effector protein, recognizes complementary RNA sequences as long as the repeatportion of the crRNA cannot extensively base pair with the target (Meeske and Marraffini, 2018). Upon target recognition, Cas13 undergoes a conformational change that activates the effector's ribonuclease domain, resulting in non-specific cleavage of the proximal portions of the target RNA (Liu et al., 2017a,b). The effector domain remains highly active even after cleavage of local RNAs, leading to the extensive degradation of cellular RNAs. The degradation can be sufficiently extensive to shut down the host's growth, resulting in a reversible dormancy state (Abudayyeh et al., 2016; Meeske et al., 2019). The activity of type VI CRISPRCas targeting also had a more severe effect on the fitness of $E$. coli during high production of target RNA, potentially allowing the cell to survive self-targeting by Cas13 if the target RNA is not highly expressed (Abudayyeh et al., 2016) and sparing the cells from self-targeting induced dormancy.

Another consequence of RNA-based (self-)targeting is type III systems and Cas13 ignoring transcriptionally silent targets. Activation of type III and VI systems only upon RNA recognition would be particularly important for temperate phages and viruses whose lytic genes are repressed during lysogeny (Johnson et al., 1981). Therefore, if a spacer directs the Csm/Cmr effector complex or Cas13 to an RNA necessary for the lytic cycle, then only the lysogens entering the lytic cycle will be targeted. Tolerance of a prophage has been shown for the type IIIA system in Staphylococcus epidermidis that actively targets its own prophages only upon transition into the lytic cycle (Goldberg et al., 2014). By only targeting phages and viruses in the lytic cycle, cells are able to maintain any potentially positive functions that might arise from a prophage/provirus encoded in their genome and prevent cell death during the invader's lytic phase.

\section{Genome-Encoded Anti-CRISPR Proteins}

Escape from targeting is not limited to genetically disrupting the CRISPR-Cas system or its target; another means involves inhibiting CRISPR-Cas activity in trans by Acrs. These proteins allow phages/viruses to thwart immunity by CRISPR-Cas systems (Pawluk et al., 2018). So far, Acrs have been identified that inhibit different subtypes of type I, II, III, and V CRISPR-Cas systems (Bondy-Denomy et al., 2013; Pawluk et al., 2014, 2016a,b; Hynes et al., 2017; Rauch et al., 2017; He et al., 2018; Marino et al., 2018; Watters et al., 2018; Bhoobalan-Chitty et al., 2019), and Acrs against type IV and VI systems likely await discovery. The Acrs identified to-date have exhibited remarkable diversity in their sequence and in their mechanism of action, such as blocking DNA binding, preventing effector complex formation, sequestering the nuclease into dimers, blocking nuclease activity or preventing nuclease recruitment (Bondy-Denomy et al., 2015; Pawluk et al., 2018; Thavalingam et al., 2019).

Acrs allow phages and viruses to not only escape attack by CRISPR-Cas systems but also protect a lysogenized phage/provirus (not to mention the host chromosome) from an endogenous CRISPR-Cas system encoding a viral-targeting or chromosomal-targeting spacer (Figure 2B). Therefore, a genome encoding both a CRISPR-Cas system and a selftargeting spacer could potentially also encode an Acr. Rauch et al. (2017) hypothesized that self-targeting spacers would indicate the presence of an inhibiting Acr, which led them to identify four Acrs encoded in prophage regions of Listeria monocytogenes that inhibit Cas9. Separately, Watters et al. (2018) and Marino et al. (2018) used a similar approach to identify Acrs in Moraxella bovoculi active against type I and type V CRISPR-Cas systems. Given the success in identifying Acrs in prokaryotes harboring self-targeting CRISPR-Cas systems, this mechanism could principally explain the natural appearance of self-targeting spacers.

\section{SELF-TARGETING SPACERS UNDERLYING ALTERNATIVE FUNCTIONS OF CRISPR-CAS SYSTEMS}

We have described how self-targeting spacers can be acquired and how cells can avoid the cytotoxic impact of self-targeting. In some of these cases, self-targeting could reflect an alternative 
function of the CRISPR-Cas system. Here, we describe different examples in which self-targeting has impacted the host or in which a mechanism has been reported that could impact host behavior, potentially foreshadowing an alternative function. These examples can be divided into four categories: genome evolution, RNA degradation, transcriptional repression, and foreign invaders co-opting self-targeting CRISPR-Cas systems. While conserved examples of CRISPR-Cas systems performing alternative functions have not been described, there has been a steady increase in anecdotal examples that suggest that CRISPRCas systems can stray from adaptive immunity, with varying benefits to the host.

\section{Genome Evolution}

One reported outcome of acquiring self-targeting spacers is genome evolution by forcing the host to mutate in order to escape autoimmunity. While this mechanism still reflects active DNA targeting through the standard steps of CRISPR-based immunity and thus may not represent a "true" alternative function, we still consider this an alternative function because of the largescale change in genomic content that can confer benefits to the host. Specifically, chromosomal targeting can lead to mutations or small deletions in the target gene. These deletions can also be much larger and encompass many surrounding non-targeted genes. While any loss of an essential gene would be lethal, these larger deletions could also provide a fitness advantage by generating new phenotypes or reducing the overall size of the genome, and remodeling of pathogenicity islands could cause a change in bacterial virulence (Vercoe et al., 2013; Westra et al., 2014). Besides triggering active mutations, self-targeting by CRISPR-Cas systems can also select for a small sub-population already lacking the target (Dy et al., 2013; Selle et al., 2015).

Self-targeting by CRISPR-Cas systems can further lead to bacterial or archaeal evolution by disrupting an important gene and forcing the organism to adapt to this change. One important example comes from the bacterium Pelobacter carbinolicus. Unlike other members of the Geobacteraceae family, $P$. carbinolicus cannot reduce $\mathrm{Fe}(\mathrm{III})$ as part of its metabolism (Richter et al., 2007). This phenotype is potentially caused by an existing spacer within the endogenous type I-E CRISPR-Cas system that is complementary to a region within the histidyl-tRNA synthetase gene hisS. A lack of histidyl-tRNA synthetase would lead to reduced translation of proteins with multiple closely spaced histidines. The hisStargeting spacer is located opposite of the end of the CRISPR array where new spacers are added, suggesting that the uptake of this spacer did not occur recently. Supporting the active targeting of hisS, transforming the self-targeting spacer and the hisS gene from $P$. carbinolicus into a genetically tractable strain of the related species Geobacter sulfurreducens resulted in few transformants, and these transformants grew poorly. $P$. carbinolicus has also lost or mutated multiple genes with high histidine content that are still present in closely related species, potentially also explaining the loss of Fe(III)-respiration (Aklujkar and Lovley, 2010). It would be interesting to see how the endogenous I-E system is impacting HisS expression without driving lethal autoimmunity, where we expect the mechanism to fall under one of the categories below.

\section{CRISPR-Cas Induced mRNA Cleavage}

Not all CRISPR-Cas systems solely target DNA, wherein RNA targeting could modulate gene expression without inducing cytotoxicity. To-date, type III, type VI, some type I, and some type II CRISPR-Cas systems have been shown to target RNA (Hale et al., 2009, 2012; O’Connell et al., 2014; Samai et al., 2015; Abudayyeh et al., 2016; Li R. et al., 2016; Dugar et al., 2018; Rousseau et al., 2018; Strutt et al., 2018). In the event that RNA but not DNA is targeted, selftargeting spacers would not necessarily result in autoimmunity but instead could degrade mRNA and lead to changes in gene expression.

The type III-B CRISPR-Cas system in Myxococcus xanthus is a potential example that degrades mRNA, although this mechanism remains to be fully established (Wallace et al., 2014). As part of the study, the authors performed a transposon screen in a $\triangle$ pilA strain lacking the type IV pilus required for exopolysaccharide production. They isolated a mutant with a transposon inserted into the CRISPR3 array, which coincided with restored exopolysaccharide production and impaired fruiting body development. Wallace et al. (2014) proposed a mechanism in which the transposon enhanced precrRNA processing, leading to crRNA-dependent regulation of exopolysaccharide production and fruiting body development. Other possibilities are that the repertoire of crRNAs includes a portion of the transposon, altering the targeting potential of the array. Given more recent reports of type III-B systems targeting transcriptionally active DNA (Peng et al., 2015; Estrella et al., 2016), other mechanisms may be at work in M. xanthus harboring the transposon insertion.

Another alternative function via self-targeting that appears to involve mRNA degradation allows the pathogen Pseudomonas aeruginosa to evade immune detection (Figure 3). The type I-F system in $P$. aeruginosa strain UCBPP-PA14 encodes one spacer within its CRISPR1 array that bears partial complementarity to the chromosomally encoded las $R$ gene. LasR is a bacterial quorum sensing regulator whose regulon includes virulence-associated factors presumably detected through Toll-like receptor 4 in mammals. The self-targeting spacer did not lead to any detectable cleavage of the chromosomal DNA but instead appeared to cleave the lasR mRNA. Downregulation of this receptor in turn led to a reduced pro-inflammatory response. The suspected target within the lasR mRNA spans 12 nts, with one internal mismatch and base pairs with the $3^{\prime}$ end of the spacer. Mutational analysis further revealed that disrupting a $5^{\prime}$-GGN-3' sequence immediately upstream of the lasR target as well as the following 8 base pairs blocked mRNA target degradation (Li R. et al., 2016).

As a brief follow-up to this study, Müller-Esparza and Randau searched for other potentially targeted mRNAs within the $P$. aeruginosa UCBPP-PA14 strain based on potential target sites that include the upstream $5^{\prime}$-GGN-3' sequence followed by nine complementary nts. They could identify 189 putative targeted 


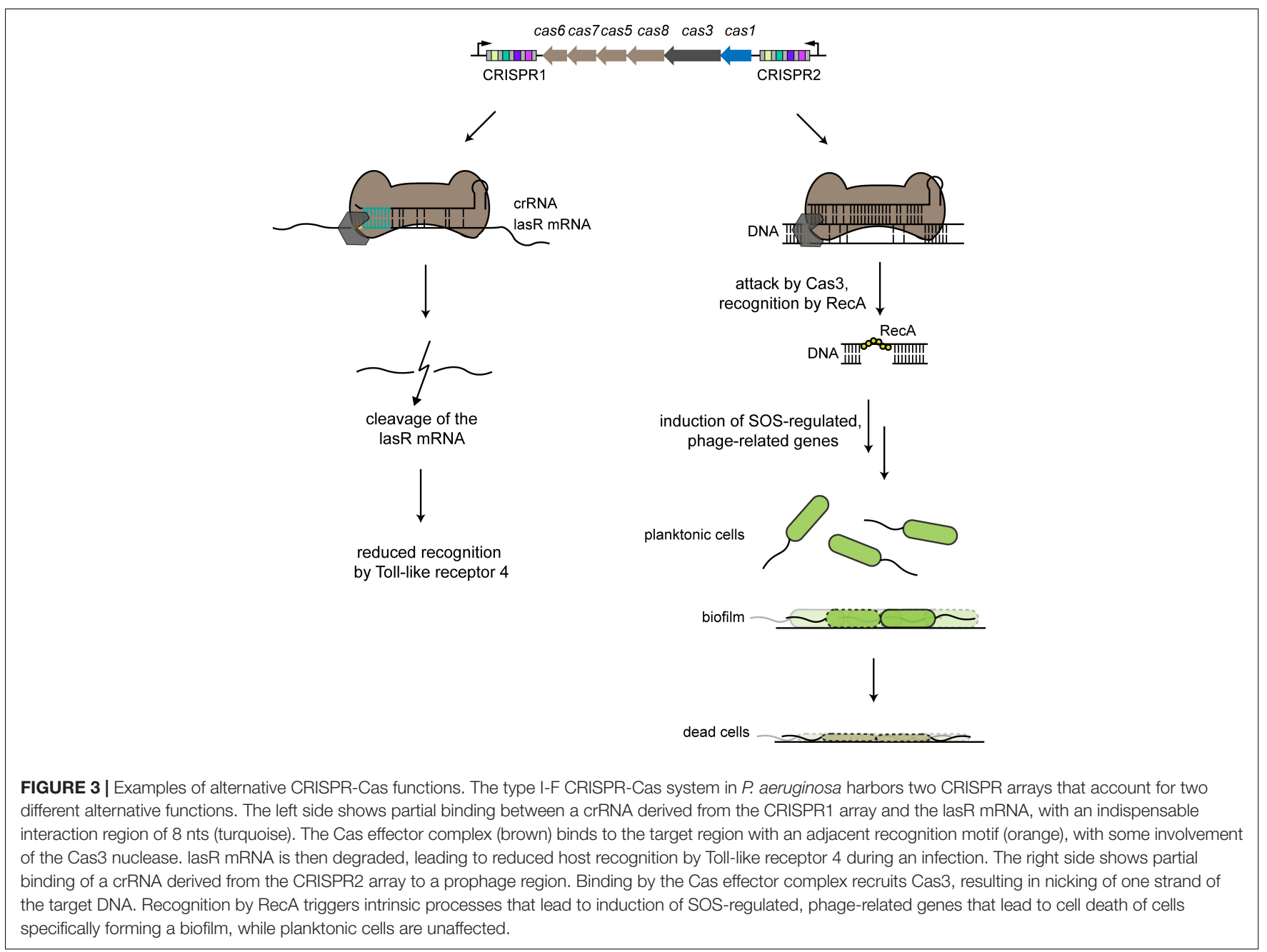

mRNAs, suggesting that additional requirements such as mRNA secondary structure are needed for mRNA targeting. Therefore, further studies are necessary to clarify the requirements for mRNA degradation by the type I-F CRISPR-Cas system in this strain of $P$. aeruginosa and the many other organisms encoding these systems (Müller-Esparza and Randau, 2017).

Cas9 is traditionally seen as a DNA-targeting nuclease, yet emerging examples have revealed that some Cas9s can also target RNA (O'Connell et al., 2014; Rousseau et al., 2018; Strutt et al., 2018). Original studies of the Cas9 from Streptococcus pyogenes suggested that the effector protein could differentiate between RNA and DNA (Gasiunas et al., 2012), wherein RNA targeting could only be achieved by hybridizing RNA with a PAM-presenting oligonucleotide (PAMmer) (O'Connell et al., 2014; Nelles et al., 2016). Later, it was shown that some Cas9 proteins can cleave RNA even in the absence of a PAMmer. Specifically the Cas9 from the type II-C system in Neisseria meningitidis was shown to cleave RNA in vitro, while Cas9 from the type II-A system in S. aureus and the type II-C system in C. jejuni were shown to cleave RNA in vitro and in vivo (Dugar et al., 2018; Rousseau et al., 2018; Strutt et al., 2018). In all of these cases, RNA targeting did not require a flanking recognition motif. In the example from $C$. jejuni, the naturally occurring spacers were shown to bind and, in some cases, drive Cas9-mediated cleavage of endogenous RNAs. These spacers only exhibited partial complementarity to their targets, and the associated DNA sequences were not flanked by recognized PAMs, preventing genome cleavage. Dugar and coworkers did not explicitly identify a phenotype associated with RNA targeting by the endogenous Cas9 (Dugar et al., 2018), although Strutt et al. (2018) demonstrated that the Cas9 from S. aureus could inhibit gene expression through programmable RNA targeting in E. coli without leading to cell death. The above mentioned examples show that some DNA targeting systems can also target RNA, with the potential for these same systems to modulate gene expression by RNA degradation in their native hosts.

\section{CRISPR-Cas Induced DNA Damage Response}

The type I-F CRISPR-Cas system in P. aeruginosa UCBPP-PA14 performs a distinct alternative function that induces the SOS response, preventing biofilm formation and impairing swarming motility (Zegans et al., 2009; Cady and O’Toole, 2011). A key 
factor was the presence of a partial match between a spacer within the CRISPR2 array and a sequence present within the lysogenized phage DSM3 (Figure 3). The authors showed that the observed phenotype was dependent not on the presence of the lysogenized phage but rather solely on the target sequence. The presence of the CRISPR-Cas system and the PAM-flanked protospacer led surface-attached cells to undergo cell death, explaining the lack of biofilm formation. The proposed mechanism-of-action involved the recruitment of Cas3 upon binding of the CascadecrRNA complex to the region of partial complementarity, which recruited RecA and activated the SOS response upon nicking of one DNA strand. Activated RecA also triggered a pathway that led to accumulation of phage-related genes that induced cell death upon surface attachment (Heussler et al., 2015). The ensuing questions are whether this same phenomenon can be found in other biofilm-forming bacteria and whether partial genome targeting can induce other phenotypes.

\section{Transcriptional Regulation}

Beyond RNA targeting, CRISPR-Cas systems have the potential to regulate transcription through partial spacer complementarity or due to the presence of an inactivated nuclease (Sampson et al., 2013, 2019; Ratner et al., 2019). Partial complementarity resulted in regulation of transcription in Francisella novicida by so-called scaRNAs (small CRISPR/Cas-associated RNAs). ScaRNAs were encoded close to the CRISPR array associated with the type II CRISPR-Cas system in F. novicida. Strictly speaking, the scaRNAbased mechanism is not dependent on a self-targeting spacer but rather on the scaRNA acting as a crRNA. Originally it was hypothesized that the scaRNA targets RNA (Sampson et al., 2013), but later it was shown that the scaRNA hybridizes with the tracrRNA and directs Cas 9 to the partially complementary $5^{\prime}$ UTR of its endogenous DNA targets. DNA binding of the target results in transcriptional repression (Ratner et al., 2019; Sampson et al., 2019). In the case of F. novicida, targeting with the scaRNAtracrRNA-Cas 9 complex resulted in transcriptional repression of four genes contributing to its virulence by facilitating evasion from immune detection. DNA cleavage by Cas 9 is prevented through only partial complementarity of the scaRNA to the target site (Ratner et al., 2019).

Aside from transcriptional repression by DNA binding near promoter regions, another means to regulate transcription is through disruption of the Cas nuclease's active site. This phenomenon can occur in type I systems that lack the effector protein Cas3 but have an intact Cascade complex (Luo et al., 2015). It is also possible to disrupt the nucleolytic activity of a Cas effector protein by mutating the active site. For example, alanine substitutions in the $\mathrm{HNH}$ and RuvC domains in the single effectors Cas9 or Cas12a result in a catalytically dead protein that can bind a target but not cleave it (Bikard et al., 2013; Qi et al., 2013; Leenay et al., 2016). While mutations that solely inactivate cleavage are much less likely than deleterious mutations to the nuclease, either means would result in CRISPR machinery that tightly binds DNA, thereby blocking transcription. Natural examples of catalytically dead CRISPR-Cas systems acting as gene regulators have not been reported, although the ease in disrupting cas 3 in the highly prevalent type I systems would suggest that nature has regularly sampled this alternative function. Screening for CRISPR-Cas systems harboring inactive nucleases and selftargeting spacers or spacers with partial complementarity to the genome might lead to the discovery of further CRISPR-based gene regulatory systems.

\section{Invaders Co-opting CRISPR-Cas Self-Targeting}

There is also evidence of foreign invaders co-opting CRISPR-Cas systems to either promote the spread of MGEs or weaken the host's adaptive immunity through self-targeting spacers. Recent publications described CRISPR-Cas systems associated with Tn7like transposons that led to spacer-directed insertion of the transposon (Peters et al., 2017; Klompe et al., 2019; Strecker et al., 2019). The transposon portion of the system generally consists of $t n s B, t s n C$, and $t n i Q$ (a $t n s D$ homolog), yet it lacks $t n s D$ and $t n s E$ normally responsible for recognition of the attachment site (Waddell and Craig, 1988, 1989). Instead, the CRISPR-Cas portion of the system, which lacks nuclease activity and the acquisition machinery, directs transposon insertion up to $\sim 80$ nts downstream of the target site. Because the target site is preserved, an integrated CRISPR transposon would inherently encode a self-targeting spacer (Klompe et al., 2019; Strecker et al., 2019). Nevertheless, the self-targeting spacer appears to be no longer functional due to the lack of multiple transposon insertions at the same target site (Strecker et al., 2019). Acquiring different spacers targeting within the bacteria's genome would allow the transposon to insert itself elsewhere in the genome, although it is not known how new spacers can be acquired due to the lack of acquisition machinery.

Beyond transposons, phages and viruses also represent types of mobile genetic elements that have co-opted CRISPR-Cas systems for their own purposes. It is reported that some phages or viruses harbor at least parts of CRISPR-Cas systems (Seed et al., 2013; Hooton and Connerton, 2014; Krupovic et al., 2015; Levasseur et al., 2016; Hudaiberdiev et al., 2017; Naser et al., 2017; Dou et al., 2018). One noteworthy example comes from the lysogenic CP8/CP30A phage in C. jejuni described earlier. This phage encodes a cas4-like gene that is responsible for spacer acquisition within the type II-C CRISPR-Cas system targeting the host's genome. The authors hypothesized that these selftargeting spacers might provide a benefit for the phage infecting C. jejuni and assist in phage-mediated escape from CRISPR attack (Hooton and Connerton, 2014). Phages and viruses could escape from the host immune system by forcing the organism to use its endogenous CRISPR-Cas system for autoimmunity rather than for attacking viral invaders. Furthermore, the organism might mutate or delete its CRISPR-Cas system to prevent cell death and with this also lose the ability to target invading phages or viruses. In total, these examples show that the host and its invaders can utilize CRISPR-Cas systems and their encoded self-targeting spacers for different purposes.

\section{CONCLUSION AND FUTURE PERSPECTIVES}

Self-targeting spacers occur surprisingly often in nature, albeit less frequently than spacers matching sequences from known 
phages, viruses or plasmids. The apparent paradox between the presence of these spacers and their presumed autoimmunity can be resolved in two general ways. These spacers could represent less frequent but important biological "accidents" that compel cells to reduce or eliminate the impact of selftargeting. Alternatively, the cells could be actively using these selftargeting spacers for other purposes that extend beyond adaptive immunity. Both have been reported in the literature, with only a few examples of the latter. However, alternative functions through self-targeting spacers represent an underexplored area of research in CRISPR biology that could yield exciting new insights and tools. Below, we describe multiple opportunities for future research to uncover further instances of alternative functions, advance our understanding of CRISPR biology and evolution, and expand the available toolbox of CRISPR technologies.

One potential focus of future work is on CRISPR-Cas systems encoding multiple self-targeting spacers or on organisms encoding multiple CRISPR-Cas systems. A few examples of bacteria and archaea encoding self-targeting spacers have been reported (Stern et al., 2010) but never explored experimentally. While these examples were categorized as non-effective targeting due to the lack of an apparent PAM, mutated adjacent repeats, extended base pairing with the repeat or lack of some cas genes, these sequences could lead to some level of targeting. For instance, CRISPR nucleases are increasingly known to recognize non-canonical PAM sequences (Leenay and Beisel, 2017), and the absence of some cas genes could still allow some functions. The accumulation of multiple self-targeting spacers would also suggest a positive selective pressure. One exception could be the disruption of all but Cas1 and Cas2, possibly resulting in acquisition without negative selection against self-targeting spacers. The occurrence of prokaryotes with multiple CRISPRCas systems suggests the possibility that some systems could fulfill the canonical CRISPR function as an adaptive immune system and the others might perform alternative functions.

Another potential focus of future work is identifying spacers exhibiting partial complementarity to the host's genome. As described above, many CRISPR-Cas systems can still bind but not cleave partially complementary targets, resulting in transcriptional repression. Partial complementarity would also allow RNA targeting by some effector proteins, potentially allowing post-transcriptional regulation of endogenous genes. Standard searches for protospacers readily exclude partially matching sequences, owing in part to the difficulty in eliminating false positives. However, regardless of the source of these spacers, partial complementarity with the genome could drive alternative functions. More work is needed to understand what types of mismatches allow different CRISPR-Cas systems to bind but not cleave their targets. This information could then be fed into search algorithms tasked with identifying targets as potential sources of CRISPR-Cas systems moonlighting as gene regulators.

Anti-CRISPR proteins could also provide a potential source for alternative functions. As described above, one strategy to find new anti-CRISPR proteins is to identify organisms with self-targeting spacers (Rauch et al., 2017; Watters et al., 2018). However, the search could be reversed: identifying organisms that harbor both Acrs and CRISPR-Cas systems as potential candidates for identifying systems exhibiting alternative functions. For instance, an encoded Acr that blocks cleavage but not binding activity of the nuclease could convert the immune system into a transcriptional regulator (Pawluk et al., 2018). Discovering new Acrs still remains a major challenge, although further discoveries will enable the search for Acrs tied to alternative functions.

Beyond the discovery of novel instances of functions extending beyond adaptive immunity, interrogating how CRISPR-Cas systems exhibit alternative functions and cope with self-targeting continues to open new biotechnological applications. For instance, the recently discovered CRISPR transposons encoding genome-targeting spacers can serve as powerful tools to insert genes (Klompe et al., 2019; Strecker et al., 2019). Genome-targeting spacers have also been used with classical CRISPR-Cas systems to generate large deletions, representing important capabilities for genome engineering and minimization (Jiang et al., 2013; Oh and van Pijkeren, 2014). As there exist other means by which cells can escape autoimmunity, steps may be necessary to ensure target deletion is the predominant mode of escape. Beyond genome editing, selftargeting with endogenous CRISPR-Cas systems can be part of programmable gene regulation. The endogenous system can be rendered cleavage-deficient while preserving DNA binding activity (Luo et al., 2015). Efforts to interrogate escape from selftargeting have also revealed that gene regulation can be achieved without altering the endogenous system, such as by employing Acrs that inhibit cleavage activity but not DNA binding or by expressing partially complementary spacers. Finally, insights into self-targeting lend to employing endogenous CRISPR-Cas systems as programmable antimicrobials. If the endogenous system is fully active, self-targeting spacers can be used to kill specific bacteria (Bikard et al., 2014; Citorik et al., 2014; Gomaa et al., 2014). If the endogenous system is inhibited by an Acr, relieving expression or activity of these Acrs could unleash lethal autoimmunity, particularly if the endogenous system acquired self-targeting spacers. Further efforts to discover and elucidate new alternative functions could inspire the next generation of CRISPR technologies, emphasizing the need to further investigate the role of self-targeting CRISPR-Cas systems.

\section{AUTHOR CONTRIBUTIONS}

FW and $\mathrm{CB}$ conceived and wrote the manuscript.

\section{FUNDING}

This work was supported by funding from the Deutsche Forschungsgemeinschaft (BE 6703/1-1 to CB).

\section{ACKNOWLEDGMENTS}

We thank Daphne Collias for critical feedback on the manuscript. 


\section{REFERENCES}

Abudayyeh, O. O., Gootenberg, J. S., Konermann, S., Joung, J., Slaymaker, I. M., Cox, D. B. T., et al. (2016). C2c2 is a single-component programmable RNAguided RNA-targeting CRISPR effector. Science 353:aaf5573. doi: 10.1126/ science.aaf5573

Akhter, S., Aziz, R. K., and Edwards, R. A. (2012). PhiSpy: a novel algorithm for finding prophages in bacterial genomes that combines similarity- and composition-based strategies. Nucleic Acids Res. 40:e126. doi: 10.1093/nar/ gks406

Aklujkar, M., and Lovley, D. R. (2010). Interference with histidyl-tRNA synthetase by a CRISPR spacer sequence as a factor in the evolution of Pelobacter carbinolicus. BMC Evol. Biol. 10:230. doi: 10.1186/1471-2148-10-230

Aravind, L., and Koonin, E. V. (2001). Prokaryotic homologs of the eukaryotic DNA-end-binding protein $\mathrm{Ku}$, novel domains in the Ku protein and prediction of a prokaryotic double-strand break repair system. Genome Res. 11, 1365-1374. doi: $10.1101 /$ gr.181001

Barrangou, R., Fremaux, C., Deveau, H., Richards, M., Boyaval, P., Moineau, S., et al. (2007). CRISPR provides acquired resistance against viruses in prokaryotes. Science 315, 1709-1712. doi: 10.1126/science.1138140

Behler, J., Sharma, K., Reimann, V., Wilde, A., Urlaub, H., and Hess, W. R. (2018). The host-encoded RNase E endonuclease as the crRNA maturation enzyme in a CRISPR-Cas subtype III-Bv system. Nat. Microbiol. 3, 367-377. doi: 10.1038/s41564-017-0103-5

Benda, C., Ebert, J., Scheltema, R. A., Schiller, H. B., Baumgärtner, M., Bonneau, F., et al. (2014). Structural model of a CRISPR RNA-silencing complex reveals the RNA-target cleavage activity in Cmr4. Mol. Cell 56, 43-54. doi: 10.1016/j. molcel.2014.09.002

Bernheim, A., Calvo-Villamañán, A., Basier, C., Cui, L., Rocha, E. P. C., Touchon, M., et al. (2017). Inhibition of NHEJ repair by type II-A CRISPR-Cas systems in bacteria. Nat. Commun. 8:2094. doi: 10.1038/s41467-017-02350-1

Bhoobalan-Chitty, Y., Johansen, T. B., Di Cianni, N., and Peng, X. (2019). Inhibition of type III CRISPR-Cas immunity by an archaeal virus-encoded anti-CRISPR protein. Cell 179:448-458.e11. doi: 10.1016/j.cell.2019.09.003

Bikard, D., Euler, C. W., Jiang, W., Nussenzweig, P. M., Goldberg, G. W., Duportet, X., et al. (2014). Exploiting CRISPR-Cas nucleases to produce sequence-specific antimicrobials. Nat. Biotechnol. 32, 1146-1150. doi: 10.1038/nbt.3043

Bikard, D., Hatoum-Aslan, A., Mucida, D., and Marraffini, L. A. (2012). CRISPR interference can prevent natural transformation and virulence acquisition during in vivo bacterial infection. Cell Host Microbe 12, 177-186. doi: 10.1016/ j.chom.2012.06.003

Bikard, D., Jiang, W., Samai, P., Hochschild, A., Zhang, F., and Marraffini, L. A. (2013). Programmable repression and activation of bacterial gene expression using an engineered CRISPR-Cas system. Nucleic Acids Res. 41, 7429-7437. doi: 10.1093/nar/gkt520

Bobay, L.-M., Touchon, M., and Rocha, E. P. C. (2013). Manipulating or superseding host recombination functions: a dilemma that shapes phage evolvability. PLoS Genetics 9:e1003825. doi: 10.1371/journal.pgen.100 3825

Bolotin, A., Quinquis, B., Renault, P., Sorokin, A., Ehrlich, S. D., Kulakauskas, S., et al. (2004). Complete sequence and comparative genome analysis of the dairy bacterium Streptococcus thermophilus. Nat. Biotechnol. 22, 1554-1558. doi: $10.1038 / \mathrm{nbt} 1034$

Bolotin, A., Quinquis, B., Sorokin, A., and Ehrlich, S. D. (2005). Clustered regularly interspaced short palindrome repeats (CRISPRs) have spacers of extrachromosomal origin. Microbiology 151, 2551-2561. doi: 10.1099/mic.0. 28048-0

Bondy-Denomy, J., Garcia, B., Strum, S., Du, M., Rollins, M. F., Hidalgo-Reyes, Y., et al. (2015). Multiple mechanisms for CRISPR-Cas inhibition by anti-CRISPR proteins. Nature 526, 136-139. doi: 10.1038/nature15254

Bondy-Denomy, J., Pawluk, A., Maxwell, K. L., and Davidson, A. R. (2013). Bacteriophage genes that inactivate the CRISPR/Cas bacterial immune system. Nature 493, 429-432. doi: 10.1038/nature11723

Bowater, R., and Doherty, A. J. (2006). Making ends meet: repairing breaks in bacterial DNA by non-homologous end-joining. PLoS Genet. 2:e8. doi: 10.1371/ journal.pgen.0020008
Brodt, A., Lurie-Weinberger, M. N., and Gophna, U. (2011). CRISPR loci reveal networks of gene exchange in archaea. Biol. Direct 6:65. doi: 10.1186/17456150-6-65

Brouns, S. J. J., Jore, M. M., Lundgren, M., Westra, E. R., Slijkhuis, R. J. H., Snijders, A. P. L., et al. (2008). Small CRISPR RNAs guide antiviral defense in prokaryotes. Science 321, 960-964. doi: 10.1126/science.1159689

Cady, K. C., and O'Toole, G. A. (2011). Non-identity-mediated CRISPR-bacteriophage interaction mediated via the Csy and Cas3 proteins. J. Bacteriol. 193, 3433-3445. doi: 10.1128/JB. 01411-10

Cañez, C., Selle, K., Goh, Y. J., and Barrangou, R. (2019). Outcomes and characterization of chromosomal self-targeting by native CRISPR-Cas systems in Streptococcus thermophilus. FEMS Microbiol. Lett. 366:fnz105. doi: 10.1093/ femsle/fnz105

Carte, J., Wang, R., Li, H., Terns, R. M., and Terns, M. P. (2008). Cas6 is an endoribonuclease that generates guide RNAs for invader defense in prokaryotes. Genes Dev. 22, 3489-3496. doi: 10.1101/gad.174 2908

Chayot, R., Montagne, B., Mazel, D., and Ricchetti, M. (2010). An end-joining repair mechanism in Escherichia coli. Proc. Natl. Acad. Sci. U.S.A. 107, $2141-$ 2146. doi: 10.1073/pnas.0906355107

Citorik, R. J., Mimee, M., and Lu, T. K. (2014). Sequence-specific antimicrobials using efficiently delivered RNA-guided nucleases. Nat. Biotechnol. 32, 11411145. doi: 10.1038/nbt.3011

Cui, L., and Bikard, D. (2016). Consequences of Cas9 cleavage in the chromosome of Escherichia coli. Nucleic Acids Res. 44, 4243-4251. doi: 10.1093/nar/gk w223

Datsenko, K. A., Pougach, K., Tikhonov, A., Wanner, B. L., Severinov, K., and Semenova, E. (2012). Molecular memory of prior infections activates the CRISPR/Cas adaptive bacterial immunity system. Nat. Commun. 3:945. doi: 10.1038/ncomms1937

Deltcheva, E., Chylinski, K., Sharma, C. M., Gonzales, K., Chao, Y., Pirzada, Z. A., et al. (2011). CRISPR RNA maturation by trans-encoded small RNA and host factor RNase III. Nature 471, 602-607. doi: 10.1038/nature 09886

Deveau, H., Barrangou, R., Garneau, J. E., Labonté, J., Fremaux, C., Boyaval, P., et al. (2008). Phage response to CRISPR-encoded resistance in Streptococcus thermophilus. J. Bacteriol. 190, 1390-1400. doi: 10.1128/jb.01412-07

Dou, C., Yu, M., Gu, Y., Wang, J., Yin, K., Nie, C., et al. (2018). Structural and mechanistic analyses reveal a unique Cas4-like protein in the mimivirus virophage resistance element system. iScience 3, 1-10. doi: 10.1016/j.isci.2018. 04.001

Dugar, G., Leenay, R. T., Eisenbart, S. K., Bischler, T., Aul, B. U., Beisel, C. L., et al. (2018). CRISPR RNA-dependent binding and cleavage of endogenous RNAs by the Campylobacter jejuni Cas9. Mol. Cell 69:893-905.e7. doi: 10.1016/j.molcel. 2018.01.032

Dy, R. L., Pitman, A. R., and Fineran, P. C. (2013). Chromosomal targeting by CRISPR-Cas systems can contribute to genome plasticity in bacteria. Mob. Genet. Elements 3:e26831. doi: 10.4161/mge.26831

El Karoui, M., Biaudet, V., Schbath, S., and Gruss, A. (1999). Characteristics of Chi distribution on different bacterial genomes. Res. Microbiol. 150, 579-587. doi: 10.1016/s0923-2508(99)00132-1

Elmore, J. R., Sheppard, N. F., Ramia, N., Deighan, T., Li, H., Terns, R. M., et al. (2016). Bipartite recognition of target RNAs activates DNA cleavage by the Type III-B CRISPR-Cas system. Genes Dev. 30, 447-459. doi: 10.1101/gad.27215 3.115

Estrella, M. A., Kuo, F.-T., and Bailey, S. (2016). RNA-activated DNA cleavage by the Type III-B CRISPR-Cas effector complex. Genes Dev. 30, 460-470. doi: $10.1101 /$ gad. 273722.115

Fineran, P. C., Gerritzen, M. J. H., Suárez-Diez, M., Künne, T., Boekhorst, J., van Hijum, S. A. F. T., et al. (2014). Degenerate target sites mediate rapid primed CRISPR adaptation. Proc. Natl. Acad. Sci. U.S.A. 111, E1629-E1638. doi: $10.1073 /$ pnas. 1400071111

Friedman, S. A., and Hays, J. B. (1986). Selective inhibition of Escherichia coli RecBC activities by plasmid-encoded GamS function of phage lambda. Gene 43, 255-263. doi: 10.1016/0378-1119(86)90214-3 
Gasiunas, G., Barrangou, R., Horvath, P., and Siksnys, V. (2012). Cas9-crRNA ribonucleoprotein complex mediates specific DNA cleavage for adaptive immunity in bacteria. Proc. Natl. Acad. Sci. U.S.A. 109, E2579-E2586.

Geer, L. Y., Marchler-Bauer, A., Geer, R. C., Han, L., He, J., He, S., et al. (2010). The NCBI BioSystems database. Nucleic Acids Res. 38, D492-D496. doi: 10.1093/ nar/gkp858

Goldberg, G. W., Jiang, W., Bikard, D., and Marraffini, L. A. (2014). Conditional tolerance of temperate phages via transcription-dependent CRISPR-Cas targeting. Nature 514, 633-637. doi: 10.1038/nature13637

Gomaa, A. A., Klumpe, H. E., Luo, M. L., Selle, K., Barrangou, R., and Beisel, C. L. (2014). Programmable removal of bacterial strains by use of genome-targeting CRISPR-Cas systems. mBio 5:e928-13. doi: 10.1128/mBio.00928-13

Gong, C., Bongiorno, P., Martins, A., Stephanou, N. C., Zhu, H., Shuman, S., et al. (2005). Mechanism of nonhomologous end-joining in mycobacteria: a lowfidelity repair system driven by Ku, ligase D and ligase C. Nat. Struct. Mol. Biol. 12, 304-312. doi: 10.1038/nsmb915

Grissa, I., Vergnaud, G., and Pourcel, C. (2007). The CRISPRdb database and tools to display CRISPRs and to generate dictionaries of spacers and repeats. BMC Bioinformatics 8:172. doi: 10.1186/1471-2105 $-8-172$

Guan, J., Wang, W., and Sun, B. (2017). Chromosomal targeting by the type IIIA CRISPR-Cas system can reshape genomes in Staphylococcus aureus. mSphere 2:e403-17. doi: 10.1128/msphere.00403-17

Hale, C. R., Majumdar, S., Elmore, J., Pfister, N., Compton, M., Olson, S., et al. (2012). Essential features and rational design of CRISPR RNAs that function with the Cas RAMP module complex to cleave RNAs. Mol. Cell 45, 292-302. doi: 10.1016/j.molcel.2011.10.023

Hale, C. R., Zhao, P., Olson, S., Duff, M. O., Graveley, B. R., Wells, L., et al. (2009). RNA-guided RNA cleavage by a CRISPR RNA-Cas protein complex. Cell 139, 945-956. doi: 10.1016/j.cell.2009.07.040

He, F., Bhoobalan-Chitty, Y., Van, L. B., Kjeldsen, A. L., Dedola, M., Makarova, K. S., et al. (2018). Anti-CRISPR proteins encoded by archaeal lytic viruses inhibit subtype I-D immunity. Nat. Microbiol. 3, 461-469. doi: 10.1038/s41564018-0120-z

Heler, R., Samai, P., Modell, J. W., Weiner, C., Goldberg, G. W., Bikard, D., et al. (2015). Cas9 specifies functional viral targets during CRISPR-Cas adaptation. Nature 519, 199-202. doi: 10.1038/nature14245

Heussler, G. E., Cady, K. C., Koeppen, K., Bhuju, S., Stanton, B. A., and O’Toole, G. A. (2015). Clustered regularly interspaced short palindromic repeat-dependent, biofilm-specific death of Pseudomonas aeruginosa mediated by increased expression of phage-related genes. mBio 6:e129-15. doi: 10.1128/ mBio.00129- 15

Heussler, G. E., and O’Toole, G. A. (2016). Friendly fire: biological functions and consequences of chromosomal targeting by CRISPR-Cas systems. J. Bacteriol. 198, 1481-1486. doi: 10.1128/JB.00086-16

Hooton, S. P. T., and Connerton, I. F. (2014). Campylobacter jejuni acquire new host-derived CRISPR spacers when in association with bacteriophages harboring a CRISPR-like Cas4 protein. Front. Microbiol. 5:744. doi: 10.3389/ fmicb.2014.00744

Horvath, P., Coûté-Monvoisin, A.-C., Romero, D. A., Boyaval, P., Fremaux, C., and Barrangou, R. (2009). Comparative analysis of CRISPR loci in lactic acid bacteria genomes. Int. J. Food Microbiol. 131, 62-70. doi: 10.1016/j.ijfoodmicro. 2008.05.030

Horvath, P., Romero, D. A., Coûté-Monvoisin, A.-C., Richards, M., Deveau, H., Moineau, S., et al. (2008). Diversity, activity, and evolution of CRISPR loci in Streptococcus thermophilus. J. Bacteriol. 190, 1401-1412. doi: 10.1128/jb.014 $15-07$

Hudaiberdiev, S., Shmakov, S., Wolf, Y. I., Terns, M. P., Makarova, K. S., and Koonin, E. V. (2017). Phylogenomics of Cas4 family nucleases. BMC Evol. Biol. 17:232. doi: 10.1186/s12862-017-1081-1

Hynes, A. P., Rousseau, G. M., Lemay, M.-L., Horvath, P., Romero, D. A., Fremaux, C., et al. (2017). An anti-CRISPR from a virulent streptococcal phage inhibits Streptococcus pyogenes Cas9. Nat. Microbiol. 2, 1374-1380. doi: 10.1038/s41564017-0004-7

Jackson, S. A., Birkholz, N., Malone, L. M., and Fineran, P. C. (2019). Imprecise spacer acquisition generates CRISPR-Cas immune diversity through primed adaptation. Cell Host Microbe 25:250-260.e4. doi: 10.1016/j.chom.2018. 12.014
Jiang, W., Bikard, D., Cox, D., Zhang, F., and Marraffini, L. A. (2013). RNA-guided editing of bacterial genomes using CRISPR-Cas systems. Nat. Biotechnol. 31, 233-239. doi: 10.1038/nbt.2508

Johnson, A. D., Poteete, A. R., Lauer, G., Sauer, R. T., Ackers, G. K., and Ptashne, M. (1981). $\lambda$ Repressor and cro-components of an efficient molecular switch. Nature 294, 217-223. doi: 10.1038/294217a0

Jung, T.-Y., An, Y., Park, K.-H., Lee, M.-H., Oh, B.-H., and Woo, E. (2015). Crystal structure of the Csm1 subunit of the Csm complex and its single-stranded DNA-specific nuclease activity. Structure 23, 782-790. doi: 10.1016/j.str.2015. 01.021

Kazlauskiene, M., Kostiuk, G., Venclovas, Č, Tamulaitis, G., and Siksnys, V. (2017). A cyclic oligonucleotide signaling pathway in type III CRISPR-Cas systems. Science 357, 605-609. doi: 10.1126/science.aao0100

Kazlauskiene, M., Tamulaitis, G., Kostiuk, G., Venclovas, Č, and Siksnys, V. (2016). Spatiotemporal control of type III-A CRISPR-Cas immunity: coupling DNA degradation with the target RNA recognition. Mol. Cell 62, 295-306. doi: 10. 1016/j.molcel.2016.03.024

Kersey, P. J., Allen, J. E., Allot, A., Barba, M., Boddu, S., Bolt, B. J., et al. (2018). Ensembl Genomes 2018: an integrated omics infrastructure for non-vertebrate species. Nucleic Acids Res. 46, D802-D808. doi: 10.1093/nar/gkx1011

Kieper, S. N., Almendros, C., Behler, J., McKenzie, R. E., Nobrega, F. L., Haagsma, A. C., et al. (2018). Cas4 facilitates PAM-compatible spacer selection during CRISPR adaptation. Cell Rep. 22, 3377-3384. doi: 10.1016/j.celrep.2018.02.103

Klompe, S. E., Vo, P. L. H., Halpin-Healy, T. S., and Sternberg, S. H. (2019). Transposon-encoded CRISPR-Cas systems direct RNA-guided DNA integration. Nature 571, 219-225. doi: 10.1038/s41586-019-1323-z

Kojima, K. K., and Kanehisa, M. (2008). Systematic survey for novel types of prokaryotic retroelements based on gene neighborhood and protein architecture. Mol. Biol. Evol. 25, 1395-1404. doi: 10.1093/molbev/msn081

Koonin, E. V., and Makarova, K. S. (2019). Origins and evolution of CRISPR-Cas systems. Philos. Trans. R. Soc. Lond. B Biol. Sci. 374:20180087. doi: 10.1098/rstb. 2018.0087

Koonin, E. V., Makarova, K. S., and Zhang, F. (2017). Diversity, classification and evolution of CRISPR-Cas systems. Curr. Opin. Microbiol. 37, 67-78. doi: 10.1016/j.mib.2017.05.008

Krupovic, M., Cvirkaite-Krupovic, V., Prangishvili, D., and Koonin, E. V. (2015). Evolution of an archaeal virus nucleocapsid protein from the CRISPRassociated Cas4 nuclease. Biol. Direct. 10:65. doi: 10.1186/s13062-015-0 093-2

Künne, T., Kieper, S. N., Bannenberg, J. W., Vogel, A. I. M., Miellet, W. R., Klein, M., et al. (2016). Cas3-derived target DNA degradation fragments fuel primed CRISPR adaptation. Mol. Cell 63, 852-864. doi: 10.1016/j.molcel.2016.07.011

Kuzminov, A. (2001). Single-strand interruptions in replicating chromosomes cause double-strand breaks. Proc. Natl. Acad. Sci. U.S.A. 98, 8241-8246. doi: 10.1073/pnas. 131009198

Lee, H., Dhingra, Y., and Sashital, D. G. (2019). The Cas4-Cas1-Cas2 complex mediates precise prespacer processing during CRISPR adaptation. eLife 8:e44248. doi: 10.7554/eLife.44248

Lee, H., Zhou, Y., Taylor, D. W., and Sashital, D. G. (2018). Cas4-dependent prespacer processing ensures high-fidelity programming of CRISPR arrays. Mol. Cell 70:48-59.e5. doi: 10.1016/j.molcel.2018.03.003

Leenay, R. T., and Beisel, C. L. (2017). Deciphering, communicating, and engineering the CRISPR PAM. J. Mol. Biol. 429, 177-191. doi: 10.1016/j.jmb. 2016.11.024

Leenay, R. T., Maksimchuk, K. R., Slotkowski, R. A., Agrawal, R. N., Gomaa, A. A., Briner, A. E., et al. (2016). Identifying and visualizing functional PAM diversity across CRISPR-Cas Systems. Mol. Cell 62, 137-147. doi: 10.1016/j.molcel.2016. 02.031

Lemak, S., Beloglazova, N., Nocek, B., Skarina, T., Flick, R., Brown, G., et al. (2013). Toroidal structure and DNA cleavage by the CRISPR-associated [4Fe4S] cluster containing Cas4 nuclease SSO0001 from Sulfolobus solfataricus. J. Am. Chem. Soc. 135, 17476-17487. doi: 10.1021/ja408729b

Levasseur, A., Bekliz, M., Chabrière, E., Pontarotti, P., La Scola, B., and Raoult, D. (2016). MIMIVIRE is a defence system in Mimivirus that confers resistance to virophage. Nature 531, 249-252. doi: 10.1038/nature17146

Levy, A., Goren, M. G., Yosef, I., Auster, O., Manor, M., Amitai, G., et al. (2015). CRISPR adaptation biases explain preference for acquisition of foreign DNA. Nature 520, 505-510. doi: 10.1038/nature14302 
Li, R., Fang, L., Tan, S., Yu, M., Li, X., He, S., et al. (2016). Type I CRISPR-Cas targets endogenous genes and regulates virulence to evade mammalian host immunity. Cell Res. 26, 1273-1287. doi: 10.1038/cr.2016.135

Li, Y., Pan, S., Zhang, Y., Ren, M., Feng, M., Peng, N., et al. (2016). Harnessing Type I and Type III CRISPR-Cas systems for genome editing. Nucleic Acids Res. 44:e34. doi: 10.1093/nar/gkv1044

Liu, L., Li, X., Ma, J., Li, Z., You, L., Wang, J., et al. (2017a). The molecular architecture for RNA-guided RNA cleavage by Cas13a. Cell 170:714-726.e10. doi: 10.1016/j.cell.2017.06.050

Liu, L., Li, X., Wang, J., Wang, M., Chen, P., Yin, M., et al. (2017b). Two distant catalytic sites are responsible for C2c2 RNase activities. Cell 168:121-134.e10. doi: 10.1016/j.cell.2016.12.031

Liu, T., Liu, Z., Ye, Q., Pan, S., Wang, X., Li, Y., et al. (2017). Coupling transcriptional activation of CRISPR-Cas system and DNA repair genes by Csa3a in Sulfolobus islandicus. Nucleic Acids Res. 45, 8978-8992. doi: 10.1093/ nar/gkx612

Liu, T., Li, Y., Wang, X., Ye, Q., Li, H., Liang, Y., et al. (2015). Transcriptional regulator-mediated activation of adaptation genes triggers CRISPR de novo spacer acquisition. Nucleic Acids Res. 43, 1044-1055. doi: 10.1093/nar/gku1383

Luo, M. L., Mullis, A. S., Leenay, R. T., and Beisel, C. L. (2015). Repurposing endogenous type I CRISPR-Cas systems for programmable gene repression. Nucleic Acids Res. 43, 674-681. doi: 10.1093/nar/gku971

Makarova, K. S., Wolf, Y. I., Alkhnbashi, O. S., Costa, F., Shah, S. A., Saunders, S. J., et al. (2015). An updated evolutionary classification of CRISPR-Cas systems. Nat. Rev. Microbiol. 13, 722-736. doi: 10.1038/nrmicro3569

Marino, N. D., Zhang, J. Y., Borges, A. L., Sousa, A. A., Leon, L. M., Rauch, B. J., et al. (2018). Discovery of widespread type I and type V CRISPR-Cas inhibitors. Science 362, 240-242. doi: 10.1126/science.aau5174

Marraffini, L. A., and Sontheimer, E. J. (2008). CRISPR interference limits horizontal gene transfer in staphylococci by targeting DNA. Science 322, 18431845. doi: $10.1126 /$ science. 1165771

Marraffini, L. A., and Sontheimer, E. J. (2010). Self versus non-self discrimination during CRISPR RNA-directed immunity. Nature 463, 568-571. doi: 10.1038/ nature 08703

Meeske, A. J., and Marraffini, L. A. (2018). RNA guide complementarity prevents self-targeting in type VI CRISPR systems. Mol. Cell 71:791-801.e3. doi: 10.1016/ j.molcel.2018.07.013

Meeske, A. J., Nakandakari-Higa, S., and Marraffini, L. A. (2019). Cas13-induced cellular dormancy prevents the rise of CRISPR-resistant bacteriophage. Nature 570, 241-245. doi: 10.1038/s41586-019-1257-5

Michel, B., Flores, M. J., Viguera, E., Grompone, G., Seigneur, M., and Bidnenko, V. (2001). Rescue of arrested replication forks by homologous recombination. Proc. Natl. Acad. Sci. U.S.A. 98, 8181-8188. doi: 10.1073/pnas.111008798

Mojica, F. J. M., Díez-Villaseñor, C., García-Martínez, J., and Soria, E. (2005). Intervening sequences of regularly spaced prokaryotic repeats derive from foreign genetic elements. J. Mol. Evol. 60, 174-182. doi: 10.1007/s00239-004$0046-3$

Müller-Esparza, H., and Randau, L. (2017). Commentary: type I CRISPR-Cas targets endogenous genes and regulates virulence to evade mammalian host immunity. Front. Microbiol. 8:319.

Murphy, K. C., and Lewis, L. J. (1993). Properties of Escherichia coli expressing bacteriophage P22 Abc (anti-RecBCD) proteins, including inhibition of Chi activity. J. Bacteriol. 175, 1756-1766. doi: 10.1128/jb.175.6.1756-1766.1993

Naser, I. B., Hoque, M. M., Nahid, M. A., Tareq, T. M., Rocky, M. K., and Faruque, S. M. (2017). Analysis of the CRISPR-Cas system in bacteriophages active on epidemic strains of Vibrio cholerae in Bangladesh. Sci. Rep. 7:14880. doi: 10.1038/s41598-017-14839-2

Nelles, D. A., Fang, M. Y., O'Connell, M. R., Xu, J. L., Markmiller, S. J., Doudna, J. A., et al. (2016). Programmable RNA tracking in live cells with CRISPR/Cas9. Cell 165, 488-496. doi: 10.1016/j.cell.2016.02.054

Nicholson, T. J., Jackson, S. A., Croft, B. I., Staals, R. H. J., Fineran, P. C., and Brown, C. M. (2019). Bioinformatic evidence of widespread priming in type I and II CRISPR-Cas systems. RNA Biol. 16, 566-576. doi: 10.1080/15476286. 2018.1509662

Niewoehner, O., Garcia-Doval, C., Rostøl, J. T., Berk, C., Schwede, F., Bigler, L., et al. (2017). Type III CRISPR-Cas systems produce cyclic oligoadenylate second messengers. Nature 548, 543-548. doi: 10.1038/nature23467
Nuñez, J. K., Kranzusch, P. J., Noeske, J., Wright, A. V., Davies, C. W., and Doudna, J. A. (2014). Cas1-Cas2 complex formation mediates spacer acquisition during CRISPR-Cas adaptive immunity. Nat. Struct. Mol. Biol. 21, 528-534. doi: $10.1038 / \mathrm{nsmb} .2820$

O'Connell, M. R., Oakes, B. L., Sternberg, S. H., East-Seletsky, A., Kaplan, M., and Doudna, J. A. (2014). Programmable RNA recognition and cleavage by CRISPR/Cas9. Nature 516, 263-266. doi: 10.1038/nature13769

Oh, J.-H., and van Pijkeren, J.-P. (2014). CRISPR-Cas9-assisted recombineering in Lactobacillus reuteri. Nucleic Acids Res. 42:e131-31. doi: 10.1093/nar/gku623

Paez-Espino, D., Morovic, W., Sun, C. L., Thomas, B. C., Ueda, K.-I., Stahl, B., et al. (2013). Strong bias in the bacterial CRISPR elements that confer immunity to phage. Nat. Commun. 4:1430. doi: 10.1038/ncomms2440

Paez-Espino, D., Sharon, I., Morovic, W., Stahl, B., Thomas, B. C., Barrangou, R., et al. (2015). CRISPR immunity drives rapid phage genome evolution in Streptococcus thermophilus. mBio 6:230. doi: 10.1128/mBio.00262-15

Pawluk, A., Amrani, N., Zhang, Y., Garcia, B., Hidalgo-Reyes, Y., Lee, J., et al. (2016a). Naturally occurring off-switches for CRISPR-Cas9. Cell 167:18291838.e9. doi: 10.1016/j.cell.2016.11.017

Pawluk, A., Staals, R. H. J., Taylor, C., Watson, B. N. J., Saha, S., Fineran, P. C., et al. (2016b). Inactivation of CRISPR-Cas systems by anti-CRISPR proteins in diverse bacterial species. Nat. Microbiol. 1:16085. doi: 10.1038/nmicrobiol.20 16.85

Pawluk, A., Bondy-Denomy, J., Cheung, V. H. W., Maxwell, K. L., and Davidson, A. R. (2014). A new group of phage anti-CRISPR genes inhibits the type I-E CRISPR-Cas system of Pseudomonas aeruginosa. mBio 5:e00896.

Pawluk, A., Davidson, A. R., and Maxwell, K. L. (2018). Anti-CRISPR: discovery, mechanism and function. Nat. Rev. Microbiol. 16, 12-17. doi: 10.1038/nrmicro. 2017.120

Peng, W., Feng, M., Feng, X., Liang, Y. X., and She, Q. (2015). An archaeal CRISPR type III-B system exhibiting distinctive RNA targeting features and mediating dual RNA and DNA interference. Nucleic Acids Res. 43, 406-417. doi: 10.1093/nar/gku1302

Peters, J. E., Makarova, K. S., Shmakov, S., and Koonin, E. V. (2017). Recruitment of CRISPR-Cas systems by Tn7-like transposons. Proc. Natl. Acad. Sci. U.S.A. 114, E7358-E7366. doi: 10.1073/pnas.1709035114

Pourcel, C., Salvignol, G., and Vergnaud, G. (2005). CRISPR elements in Yersinia pestis acquire new repeats by preferential uptake of bacteriophage DNA, and provide additional tools for evolutionary studies. Microbiology 151, 653-663. doi: $10.1099 /$ mic. $0.27437-0$

Qi, L. S., Larson, M. H., Gilbert, L. A., Doudna, J. A., Weissman, J. S., Arkin, A. P., et al. (2013). Repurposing CRISPR as an RNA-guided platform for sequencespecific control of gene expression. Cell 152, 1173-1183. doi: 10.1016/j.cell.2013. 02.022

Ratner, H. K., Escalera-Maurer, A., Le Rhun, A., Jaggavarapu, S., Wozniak, J. E., Crispell, E. K., et al. (2019). Catalytically active Cas9 mediates transcriptional interference to facilitate bacterial virulence. Mol. Cell 75:498-510.e5. doi: 10. 1016/j.molcel.2019.05.029

Rauch, B. J., Silvis, M. R., Hultquist, J. F., Waters, C. S., McGregor, M. J., Krogan, N. J., et al. (2017). Inhibition of CRISPR-Cas9 with bacteriophage proteins. Cell 168:150-158.e10. doi: 10.1016/j.cell.2016.12.009

Richter, C., Dy, R. L., McKenzie, R. E., Watson, B. N. J., Taylor, C., Chang, J. T., et al. (2014). Priming in the Type I-F CRISPR-Cas system triggers strandindependent spacer acquisition, bi-directionally from the primed protospacer. Nucleic Acids Res. 42, 8516-8526. doi: 10.1093/nar/gku527

Richter, H., Lanthier, M., Nevin, K. P., and Lovley, D. R. (2007). Lack of electricity production by Pelobacter carbinolicus indicates that the capacity for Fe(III) oxide reduction does not necessarily confer electron transfer ability to fuel cell anodes. Appl. Environ. Microbiol. 73, 5347-5353. doi: 10.1128/aem.00804-07

Rouillon, C., Athukoralage, J. S., Graham, S., Grüschow, S., and White, M. F. (2018). Control of cyclic oligoadenylate synthesis in a type III CRISPR system. eLife 7:e36734.

Rousseau, B. A., Hou, Z., Gramelspacher, M. J., and Zhang, Y. (2018). Programmable RNA cleavage and recognition by a natural CRISPR-Cas9 system from Neisseria meningitidis. Mol. Cell 69:906-914.e4. doi: 10.1016/j. molcel.2018.01.025

Samai, P., Pyenson, N., Jiang, W., Goldberg, G. W., Hatoum-Aslan, A., and Marraffini, L. A. (2015). Co-transcriptional DNA and RNA Cleavage during 
Type III CRISPR-Cas Immunity. Cell 161, 1164-1174. doi: 10.1016/j.cell.2015. 04.027

Sampson, T. R., Saroj, S. D., Llewellyn, A. C., Tzeng, Y.-L., and Weiss, D. S. (2013). A CRISPR/Cas system mediates bacterial innate immune evasion and virulence. Nature 497, 254-257. doi: 10.1038/nature12048

Sampson, T. R., Saroj, S. D., Llewellyn, A. C., Tzeng, Y.-L., and Weiss, D. S. (2019). Author Correction: a CRISPR/Cas system mediates bacterial innate immune evasion and virulence. Nature 570, E30-E31. doi: 10.1038/s41586-019-1253-9

Schmidt, F., Cherepkova, M. Y., and Platt, R. J. (2018). Transcriptional recording by CRISPR spacer acquisition from RNA. Nature 562, 380-385. doi: 10.1038/ s41586-018-0569- 1

Seed, K. D., Lazinski, D. W., Calderwood, S. B., and Camilli, A. (2013). A bacteriophage encodes its own CRISPR/Cas adaptive response to evade host innate immunity. Nature 494, 489-491. doi: 10.1038/nature11927

Selle, K., Klaenhammer, T. R., and Barrangou, R. (2015). CRISPR-based screening of genomic island excision events in bacteria. Proc. Natl. Acad. Sci. U.S.A. 112, 8076-8081. doi: 10.1073/pnas.1508525112

Shmakov, S., Abudayyeh, O. O., Makarova, K. S., Wolf, Y. I., Gootenberg, J. S., Semenova, E., et al. (2015). Discovery and functional characterization of diverse class 2 CRISPR-Cas systems. Mol. Cell 60, 385-397. doi: 10.1016/j.molcel.2015. 10.008

Shmakov, S. A., Sitnik, V., Makarova, K. S., Wolf, Y. I., Severinov, K. V., and Koonin, E. V. (2017). The CRISPR spacer space is dominated by sequences from species-specific mobilomes. mBio 8:e1397-17. doi: 10.1128/mBio.01397-17

Shuman, S., and Glickman, M. S. (2007). Bacterial DNA repair by non-homologous end joining. Nat. Rev. Microbiol. 5, 852-861.

Silas, S., Makarova, K. S., Shmakov, S., Páez-Espino, D., Mohr, G., Liu, Y., et al. (2017). On the origin of reverse transcriptase-using CRISPR-Cas systems and their hyperdiverse, enigmatic spacer repertoires. mBio 8:e897-17. doi: 10.1128/ mBio.00897-17

Silas, S., Mohr, G., Sidote, D. J., Markham, L. M., Sanchez-Amat, A., Bhaya, D., et al. (2016). Direct CRISPR spacer acquisition from RNA by a natural reverse transcriptase-Cas1 fusion protein. Science 351:aad4234. doi: 10.1126/science. aad 4234

Simon, D. M., and Zimmerly, S. (2008). A diversity of uncharacterized reverse transcriptases in bacteria. Nucleic Acids Res. 36, 7219-7229. doi: 10.1093/nar/ gkn867

Smith, G. R. (2012). How RecBCD enzyme and Chi promote DNA break repair and recombination: a molecular biologist's view. Microbiol. Mol. Biol. Rev. 76, 217-228. doi: 10.1128/MMBR.05026-11

Sorek, R., Lawrence, C. M., and Wiedenheft, B. (2013). CRISPR-mediated adaptive immune systems in bacteria and archaea. Annu. Rev. Biochem. 82, 237-266. doi: 10.1146/annurev-biochem-072911-172315

Staals, R. H. J., Jackson, S. A., Biswas, A., Brouns, S. J. J., Brown, C. M., and Fineran, P. C. (2016). Interference-driven spacer acquisition is dominant over naive and primed adaptation in a native CRISPR-Cas system. Nat. Commun. 7:12853.

Stachler, A.-E., Turgeman-Grott, I., Shtifman-Segal, E., Allers, T., Marchfelder, A., and Gophna, U. (2017). High tolerance to self-targeting of the genome by the endogenous CRISPR-Cas system in an archaeon. Nucleic Acids Res. 45, 5208-5216. doi: 10.1093/nar/gkx150

Stern, A., Keren, L., Wurtzel, O., Amitai, G., and Sorek, R. (2010). Self-targeting by CRISPR: gene regulation or autoimmunity? Trends Genetics 26, 335-340. doi: 10.1016/j.tig.2010.05.008

Strecker, J., Ladha, A., Gardner, Z., Schmid-Burgk, J. L., Makarova, K. S., Koonin, E. V., et al. (2019). RNA-guided DNA insertion with CRISPRassociated transposases. Science 365, 48-53. doi: 10.1126/science. aax9181

Strutt, S. C., Torrez, R. M., Kaya, E., Negrete, O. A., and Doudna, J. A. (2018). RNAdependent RNA targeting by CRISPR-Cas9. eLife 7:e32724. doi: 10.7554/eLife. 32724

Su, T., Liu, F., Chang, Y., Guo, Q., Wang, J., Wang, Q., et al. (2019). The phage T4 DNA ligase mediates bacterial chromosome DSBs repair as single component non-homologous end joining. Synth Syst. Biotechnol. 4, 107-112. doi: 10.1016/ j.synbio.2019.04.001

Swarts, D. C., Mosterd, C., van Passel, M. W. J., and Brouns, S. J. J. (2012). CRISPR interference directs strand specific spacer acquisition. PLoS One 7:e35888. doi: 10.1371/journal.pone.0035888
Tamulaitis, G., Kazlauskiene, M., Manakova, E., Venclovas, Č, Nwokeoji, A. O., Dickman, M. J., et al. (2014). Programmable RNA shredding by the type IIIA CRISPR-Cas system of Streptococcus thermophilus. Mol. Cell 56, 506-517. doi: 10.1016/j.molcel.2014.09.027

Thavalingam, A., Cheng, Z., Garcia, B., Huang, X., Shah, M., Sun, W., et al. (2019). Inhibition of CRISPR-Cas9 ribonucleoprotein complex assembly by anti-CRISPR AcrIIC2. Nat. Commun. 10:2806. doi: 10.1038/s41467-019-10 577-3

Tong, Y., Charusanti, P., Zhang, L., Weber, T., and Lee, S. Y. (2015). CRISPR-Cas9 based engineering of actinomycetal genomes. ACS Synth. Biol. 4, 1020-1029. doi: 10.1021/acssynbio.5b00038

Toro, N., Martínez-Abarca, F., Mestre, M. R., and González-Delgado, A. (2019a). Multiple origins of reverse transcriptases linked to CRISPR-Cas systems. RNA Biol. 16, 1486-1493. doi: 10.1080/15476286.2019.1639310

Toro, N., Mestre, M. R., Martínez-Abarca, F., and González-Delgado, A. (2019b). Recruitment of reverse transcriptase-Cas1 fusion proteins by Type VI-A CRISPR-Cas systems. Front. Microbiol. 10:2160. doi: 10.3389/fmicb.2019.02160

Toro, N., and Nisa-Martínez, R. (2014). Comprehensive phylogenetic analysis of bacterial reverse transcriptases. PLoS One 9:e114083. doi: 10.1371/journal.pone. 0114083

Vercoe, R. B., Chang, J. T., Dy, R. L., Taylor, C., Gristwood, T., Clulow, J. S., et al. (2013). Cytotoxic chromosomal targeting by CRISPR/Cas systems can reshape bacterial genomes and expel or remodel pathogenicity islands. PLoS Genet. 9:e1003454. doi: 10.1371/journal.pgen.1003454

Waddell, C. S., and Craig, N. L. (1988). Tn7 transposition: two transposition pathways directed by five Tn7-encoded genes. Genes Dev. 2, 137-149. doi: 10.1101/gad.2.2.137

Waddell, C. S., and Craig, N. L. (1989). Tn7 transposition: recognition of the attTn7 target sequence. Proc. Natl. Acad. Sci. U.S.A. 86, 3958-3962. doi: 10.1073/pnas. 86.11 .3958

Wallace, R. A., Black, W. P., Yang, X., and Yang, Z. (2014). A CRISPR with roles in Myxococcus xanthus development and exopolysaccharide production. J. Bacteriol. 196, 4036-4043. doi: 10.1128/JB.02035-14

Wang, J., Li, J., Zhao, H., Sheng, G., Wang, M., Yin, M., et al. (2015). Structural and mechanistic basis of PAM-dependent spacer acquisition in CRISPR-Cas systems. Cell 163, 840-853. doi: 10.1016/j.cell.2015.10.008

Watters, K. E., Fellmann, C., Bai, H. B., Ren, S. M., and Doudna, J. A. (2018). Systematic discovery of natural CRISPR-Cas12a inhibitors. Science 362, 236239. doi: 10.1126/science.aau5138

Wei, Y., Terns, R. M., and Terns, M. P. (2015). Cas9 function and host genome sampling in Type II-A CRISPR-Cas adaptation. Genes Dev. 29, 356-361. doi: 10.1101/gad.257550.114

Weller, G. R., Kysela, B., Roy, R., Tonkin, L. M., Scanlan, E., Della, M., et al. (2002). Identification of a DNA nonhomologous end-joining complex in bacteria. Science 297, 1686-1689. doi: 10.1126/science. 1074584

Westra, E. R., Buckling, A., and Fineran, P. C. (2014). CRISPR-Cas systems: beyond adaptive immunity. Nat. Rev. Microbiol. 12, 317-326. doi: 10.1038/ nrmicro3241

Wu, X., Scott, D. A., Kriz, A. J., Chiu, A. C., Hsu, P. D., Dadon, D. B., et al. (2014). Genome-wide binding of the CRISPR endonuclease Cas9 in mammalian cells. Nat. Biotechnol. 32, 670-676. doi: 10.1038/nbt.2889

Xu, T., Li, Y., Shi, Z., Hemme, C. L., Li, Y., Zhu, Y., et al. (2015). Efficient genome editing in Clostridium cellulolyticum via CRISPR-Cas9 Nickase. Appl. Environ. Microbiol. 81, 4423-4431. doi: 10.1128/AEM.00873-15

Yosef, I., Goren, M. G., and Qimron, U. (2012). Proteins and DNA elements essential for the CRISPR adaptation process in Escherichia coli. Nucleic Acids Res. 40, 5569-5576. doi: 10.1093/nar/gks216

Yosef, I., Shitrit, D., Goren, M. G., Burstein, D., Pupko, T., and Qimron, U. (2013). DNA motifs determining the efficiency of adaptation into the Escherichia coli CRISPR array. Proc. Natl. Acad. Sci. U.S.A. 110, 14396-14401. doi: 10.1073/ pnas. 1300108110

Zegans, M. E., Wagner, J. C., Cady, K. C., Murphy, D. M., Hammond, J. H., and O'Toole, G. A. (2009). Interaction between bacteriophage DMS3 and host CRISPR region inhibits group behaviors of Pseudomonas aeruginosa. J. Bacteriol. 191, 210-219. doi: 10.1128/JB.00797-08

Zerulla, K., Chimileski, S., Näther, D., Gophna, U., Thane Papke, R., and Soppa, J. (2014). DNA as a phosphate storage polymer and the alternative advantages 
of polyploidy for growth or survival. PLoS One 9:e94819. doi: 10.1371/journal. pone.0094819

Zetsche, B., Gootenberg, J. S., Abudayyeh, O. O., Slaymaker, I. M., Makarova, K. S., Essletzbichler, P., et al. (2015). Cpf1 is a single RNA-guided endonuclease of a class 2 CRISPR-Cas system. Cell 163, 759-771. doi: 10.1016/j.cell.2015.0 9.038

Zhang, F., Zhao, S., Ren, C., Zhu, Y., Zhou, H., Lai, Y., et al. (2018). CRISPRminer is a knowledge base for exploring CRISPR-Cas systems in microbe and phage interactions. Commun. Biol. 1:180. doi: 10.1038/s42003-0180184-6

Zhang, J., Kasciukovic, T., and White, M. F. (2012). The CRISPR associated protein Cas4 Is a 5' to 3' DNA exonuclease with an ironsulfur cluster. PLoS One 7:e47232. doi: 10.1371/journal.pone.004 7232
Zhang, Z., Pan, S., Liu, T., Li, Y., and Peng, N. (2019). Cas4 nucleases can effect specific integration of CRISPR spacers. J. Bacteriol. 201:e747-18. doi: 10.1128/ JB.00747-18

Conflict of Interest: The authors declare that the research was conducted in the absence of any commercial or financial relationships that could be construed as a potential conflict of interest.

Copyright (c) 2020 Wimmer and Beisel. This is an open-access article distributed under the terms of the Creative Commons Attribution License (CC BY). The use, distribution or reproduction in other forums is permitted, provided the original author(s) and the copyright owner(s) are credited and that the original publication in this journal is cited, in accordance with accepted academic practice. No use, distribution or reproduction is permitted which does not comply with these terms. 